\title{
Ground state and low-temperature magnetism of the quasi-two-dimensional honeycomb compound $\mathrm{InCu}_{2 / 3} \mathrm{~V}_{1 / 3} \mathrm{O}_{3}$
}

\author{
M. Iakovleva $\odot,{ }^{1,2,3}$ O. Janson $\odot,{ }^{1}$ H.-J. Grafe, ${ }^{1}$ A. P. Dioguardi $\odot,{ }^{1}$ H. Maeter, ${ }^{2}$ N. Yeche, ${ }^{2}$ H.-H. Klauss $\odot,{ }^{2}$ G. Pascua, ${ }^{4}$ \\ H. Luetkens, ${ }^{4}$ A. Möller ${ }^{5}{ }^{5}$ B. Büchner, ${ }^{1,2,6}$ V. Kataev $\left(\mathbb{0},{ }^{1}\right.$ and E. Vavilova ${ }^{3}$ \\ ${ }^{1}$ Leibniz Institute for Solid State and Materials Research IFW Dresden, 01171 Dresden, Germany \\ ${ }^{2}$ Institute for Solid State and Materials Physics, TU Dresden, 01069 Dresden, Germany \\ ${ }^{3}$ Zavoisky Physical-Technical Institute, FRC Kazan Scientific Center of RAS, 420029 Kazan, Russia \\ ${ }^{4}$ Labor für Myonenspinspektroskopie, Paul Scherrer Institut, CH-5232 Villigen PSI, Switzerland \\ ${ }^{5}$ JGU Mainz, 55122 Mainz, Germany \\ ${ }^{6}$ Würzburg-Dresden Cluster of Excellence ct.qmat, 97074 Würzburg, Germany
}

(Received 10 July 2019; revised manuscript received 10 October 2019; published 29 October 2019)

\begin{abstract}
We report a combined ${ }^{115} \mathrm{In}$ nuclear quadrupole resonance, ${ }^{51} \mathrm{~V}$ nuclear magnetic resonance, and muon spin-relaxation spectroscopic study of the low-temperature magnetic properties of $\mathrm{InCu}_{2 / 3} \mathrm{~V}_{1 / 3} \mathrm{O}_{3}$, a quasitwo-dimensional (2D) compound comprising in the spin sector a honeycomb lattice of antiferromagnetically coupled spins $S=1 / 2$ associated with $\mathrm{Cu}^{2+}$ ions. Despite substantial experimental and theoretical efforts, the ground state of this material has not been ultimately identified. In particular, two characteristic temperatures of about 40 and $20 \mathrm{~K}$ manifesting themselves as anomalies in different magnetic measurements are discussed controversially. A combined analysis of the experimental data complemented with theoretical calculations of exchange constants enabled us to identify, below $39 \mathrm{~K}$, an "intermediate" quasi-2D static spin state. This spin state is characterized by a staggered magnetization with a temperature evolution that agrees with the predictions for the 2D $X Y$ model. We observe that this state gradually transforms at $15 \mathrm{~K}$ into a fully developed 3D antiferromagnetic Néel state. We ascribe such an extended quasi-2D static regime to an effective magnetic decoupling of the honeycomb planes due to a strong frustration of the interlayer exchange interactions, which inhibits long-range spin-spin correlations across the planes. Interestingly, we find indications of the topological Berezinsky-Kosterlitz-Thouless transition in the quasi-2D static state of the honeycomb spin-1/2 planes of $\mathrm{InCu}_{2 / 3} \mathrm{~V}_{1 / 3} \mathrm{O}_{3}$.
\end{abstract}

DOI: 10.1103/PhysRevB.100.144442

\section{INTRODUCTION}

Dimensionality of the spin system and the involved spin degrees of freedom play an important role in defining the magnetic properties of a material, in particular regarding its ground state and magnetic excitations. Long-range magnetic order (LRO), which is a typical ground state of three-dimensional (3D) magnets, can be strongly suppressed in systems with frustrated exchange interactions. Reduction of the spatial dimensionality of magnetic couplings may be a further cause of the suppression of LRO. For instance, according to the Mermin-Wagner's theorem, LRO is impossible even at zero temperature in a $1 \mathrm{D}$ spin- $1 / 2$ isotropic Heisenberg chain with the coordination number $z=2$ due to strong quantum fluctuations, although it can be stabilized on a $2 \mathrm{D}$ square lattice with $z=4$ at $T=0 \mathrm{~K}$ [1]. The spin$1 / 2$ Heisenberg honeycomb lattice has a particular position in this respect. Like the square lattice, it is not frustrated for the antiferromagnetic (AFM) nearest-neighbor interaction. However, quantum fluctuations are enhanced due to the low coordination number $z=3$. Still, they remain less pronounced than in the 1D case and, hence, do not destroy LRO at $T=0$.

In real 3D transition-metal (TM) compounds where magnetic TM ions bonded via ligands form 2D hexagonal layers, such honeycomb spin planes may deviate from the ideal Heisenberg regime. The anisotropy of the in-plane magnetic exchange as well as a finite interlayer coupling can weaken quantum fluctuations. As a result, long-range magnetic order can be stabilized at finite temperatures [2-6]. Depending on the particular details, such as frustration of the next-neighboring in-plane exchange interaction and/or of the coupling between the planes, different types of magnetic structures can be realized [7-12]. If frustration is sufficiently strong, the LRO can be suppressed completely $[13,14]$.

One particularly interesting aspect of this class of materials is that the spin-orbit coupling and the low-symmetry ligand field can give rise to a strong easy-plane magnetic anisotropy of the honeycomb planes made of TM ions, which then can be described by the $X Y$ model. There, unlike in the Heisenberg model, only two components of the spin $S_{x}$ and $S_{y}$ are interacting on the honeycomb spin plane. This can lead to complex magnetic behavior beyond conventional LRO and associated classical spin-wave excitations, namely, the formation of the spin vortex gas and the Berezinsky-KosterlitzThouless (BKT) transition to the topologically ordered state of vortex-antivortex pairs predicted in the 2D XY model [15-18].

Only a few antiferromagnets are known so far, where the signatures of this topological transition were found mostly by 
analyzing the critical exponents. In the majority of the cases, the comparison with the predictions of the BKT theory is complicated due to the residual interplane couplings which eventually yield 3D AFM Néel order, such as in $\mathrm{BaNi}_{2}\left(\mathrm{PO}_{4}\right)_{2}$ [19], $\mathrm{BaNi}_{2} \mathrm{~V}_{2} \mathrm{O}_{8}$ [20-22], or $\mathrm{MnPS}_{3}$ [23]. As a rare exception, the coordination polymer $\mathrm{C}_{36} \mathrm{H}_{48} \mathrm{Cu}_{2} \mathrm{~F}_{6} \mathrm{~N}_{8} \mathrm{O}_{12} \mathrm{~S}_{2}$ does not exhibit the Néel order, but features magnetic excitations consistent with a BKT scenario [24].

The title compound of the present paper, $\mathrm{InCu}_{2 / 3} \mathrm{~V}_{1 / 3} \mathrm{O}_{3}$, features 2D structural order of magnetic $\mathrm{Cu}^{2+}(S=1 / 2)$ ions on a honeycomb lattice with nonmagnetic $\mathrm{V}$ ions in the formal oxidation state 5+ complementing the hexagonal layer [25]. Occasional $\mathrm{Cu} / \mathrm{V}$ site inversion gives rise to a finite structural in-plane correlation length of approximately $300 \AA$ [25]. Besides being a candidate to host $d$-wave superconductivity upon doping [26], it also appears to be a rare realization of a honeycomb spin lattice with strongly frustrated interlayer coupling. Despite substantial experimental and theoretical interest in this compound, its ground state is still not unambiguously identified. Previous magnetization, electron spin resonance (ESR), and nuclear magnetic resonance (NMR) measurements complemented by quantum Monte Carlo calculations suggest the onset of the AFM ordered state at $T_{\mathrm{N}}=38 \mathrm{~K}$ [27] with the fully developed Néel-type collinear AFM sublattices below $\sim 20 \mathrm{~K}$ [28] that feature anomalous spin dynamics [29]. However, according to Ref. [25], specificheat, thermal-expansion, and neutron-diffraction experiments show no evidence for long-range magnetic order down to $1.8 \mathrm{~K}$. As reported in Ref. [30], doping of $\mathrm{InCu}_{2 / 3} \mathrm{~V}_{1 / 3} \mathrm{O}_{3}$ with Co leads to AFM LRO, whereas $\mathrm{Zn}$ doping results in the suppression of AFM order. Theoretical studies have shown that fluctuations arising from the interlayer magnetic frustration destroy 3D magnetic $\mathrm{LRO}$ in $\mathrm{InCu}_{2 / 3} \mathrm{~V}_{1 / 3} \mathrm{O}_{3}[30,31]$.

Considering the above-mentioned controversies of different kinds of experimental data and their interpretation, it is appealing to resolve them and to obtain a unified picture of the ground state and low-temperature magnetism of $\mathrm{InCu}_{2 / 3} \mathrm{~V}_{1 / 3} \mathrm{O}_{3}$. With this aim, in the present work we investigate experimentally and theoretically the ground state and low-temperature static and dynamic properties of $\mathrm{InCu}_{2 / 3} \mathrm{~V}_{1 / 3} \mathrm{O}_{3}$. In particular, employing ${ }^{115} \mathrm{In}$ nuclear quadrupole resonance (NQR), ${ }^{51} \mathrm{~V}$ NMR, and muon spinrelaxation $(\mu \mathrm{SR})$ spectroscopies, we address the occurrence of the above-mentioned two characteristic temperature scales of $\sim 40$ and $\sim 20 \mathrm{~K}$. Their understanding appears to be crucial for the elucidation of the low-temperature magnetism of this compound. We find that below $39 \mathrm{~K}, \mathrm{InCu}_{2 / 3} \mathrm{~V}_{1 / 3} \mathrm{O}_{3}$ develops a staggered magnetization. Its $T$ dependence suggests a quasi2D static state of the spin system with predominantly in-plane commensurate long-range spin-spin correlations. Further on, we find that a full 3D antiferromagnetic order develops at a much lower temperature of $15 \mathrm{~K}$. These experimental findings are rationalized within a microscopic spin model based on density functional theory (DFT) calculations. Its key features are (i) the dominance of $J_{1}$ and the irrelevance of magnetic exchanges beyond the nearest neighbors within the honeycomb planes, i.e., $J_{1} \gg \max \left(\left|J_{2}\right|,\left|J_{3}\right|, \ldots\right)$, (ii) the $X X Z$ anisotropy of the nearest-neighbor exchange accompanied by a vanishingly small antisymmetric (Dzyaloshinskii-
Moriya) anisotropy, and (iii) the presence of a single frustrated antiferromagnetic exchange between the honeycomb planes. We argue that a significant frustration of the interlayer exchange in $\mathrm{InCu}_{2 / 3} \mathrm{~V}_{1 / 3} \mathrm{O}_{3}$, together with a certain degree of structural disorder, are responsible for the two characteristic low-temperature regimes of this compound in compliance with all experimental observations. Furthermore, by analyzing our static and dynamic magnetic data, we find indications of a BKT transition in $\mathrm{InCu}_{2 / 3} \mathrm{~V}_{1 / 3} \mathrm{O}_{3}$ presumably occurring within an intermediate quasi-2D static state of the spin system below $39 \mathrm{~K}$.

The paper is organized as follows. Experimental details are summarized in Sec. II. The results of ${ }^{115} \mathrm{In}$ NQR, ${ }^{51} \mathrm{~V}$ NMR, and $\mu$ SR experiments and the microscopic model are presented in Secs. III and IV, respectively, and discussed together in Sec. V. The main conclusions are summarized in Sec. VI. The Appendix presents some specific details of the analysis of the NQR Hamiltonian.

\section{EXPERIMENTAL DETAILS}

The powder sample of $\mathrm{InCu}_{2 / 3} \mathrm{~V}_{1 / 3} \mathrm{O}_{3}$ was synthesized and comprehensively characterized as described in Ref. [25].

NQR experiments were performed on the ${ }^{115}$ In nuclei (nuclear spin $I=9 / 2$, gyromagnetic ratio $\gamma=9.32 \mathrm{MHz} / \mathrm{T}$, quadrupolar moment $Q=0.81$ barns) using the Redstone console and Lap NMR portable spectrometers from Tecmag. Frequency-swept spectra were recorded with the $\pi / 2-\tau-$ $\pi$ pulse sequence; the spin-echo signal was integrated at each step. Measurements were performed in the temperature range from 5 to $100 \mathrm{~K}$. The nuclear relaxation rates in the NQR experiment were measured at the maximum of the spectra with the saturation-recovery method. For that, the $(9 \times \pi / 2)-\tau_{d}-\pi / 2-\pi$ pulse train was used to saturate the nuclear spin system and the echo intensity was measured as a function of the time delay $\tau_{d}$ between the pulses. The ${ }^{51} \mathrm{~V}$ NMR spin-lattice relaxation rate was measured with the method of stimulated echo.

A $\mu$ SR experiment on a powder sample of $\mathrm{InCu}_{2 / 3} \mathrm{~V}_{1 / 3} \mathrm{O}_{3}$ was performed at the GPS beam line at the Paul Scherrer Institute in Villigen, Switzerland, between 2 and $60 \mathrm{~K}$ in zero field (ZF) and $50 \mathrm{G}$ external magnetic field applied perpendicular to the forward/backward positron detector pair axis. The time-dependent corrected asymmetry spectra were analyzed using the MSRFIT software package [32].

\section{RESULTS}

\section{A. ${ }^{115}$ In NQR experiments 1. ${ }^{115}$ In NQR spectra}

The choice of the NQR technique has several advantages over the experimental methods previously used for the studies of $\mathrm{InCu}_{2 / 3} \mathrm{~V}_{1 / 3} \mathrm{O}_{3}$. As with other magnetic resonance spectroscopies, it is a local technique that provides information on the static and dynamic properties of a material on the scale of a few interatomic spacings and does not require long-range order to probe the spin structure and magnetic excitations, which would be a necessary condition, e.g., in the case of neutron scattering. Compared to NMR, NQR is a technique where no external magnetic field is needed; therefore, it 


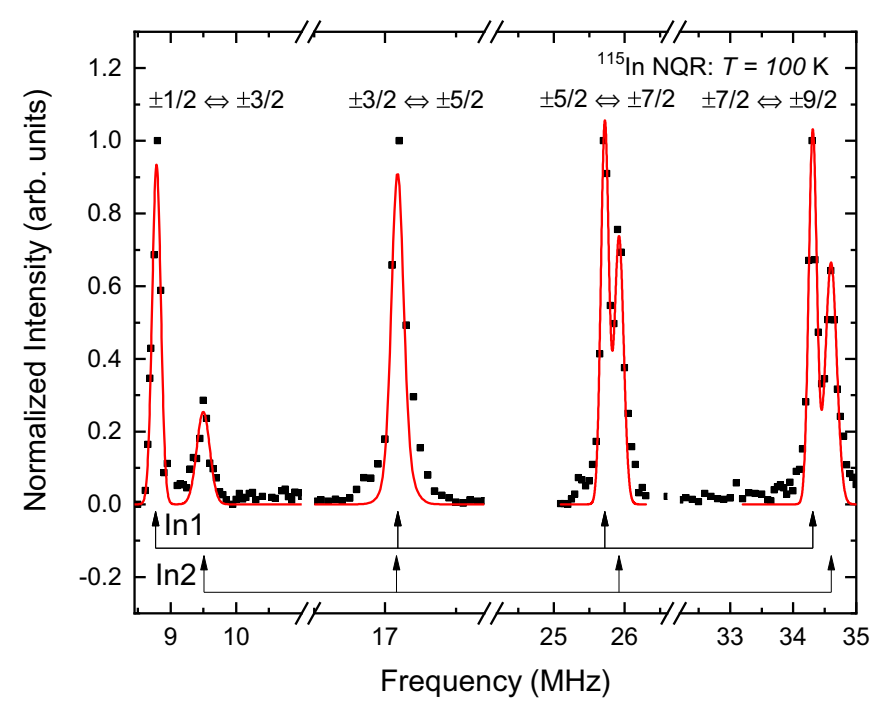

FIG. 1. High-temperature ${ }^{115} \mathrm{In} \mathrm{NQR}$ spectrum of $\mathrm{InCu}_{2 / 3} \mathrm{~V}_{1 / 3} \mathrm{O}_{3}$ (data points). The solid line is a fit to the data according to Hamiltonian (1). The positions of the lines corresponding to two nonequivalent In sites in the crystal structure (In1 and In2) are indicated by arrows (see the text for details). For technical reasons, all pairs of transitions corresponding to In 1 and In 2 were measured separately and the intensities of the respective parts of the spectrum were each normalized to unity.

allows one to probe the bare, unperturbed Hamiltonian of the electron spin system. Finally, NQR is very sensitive not only to fluctuating and static local magnetic fields generated by electron spins, but also to the electric-field gradient around the nuclei, thus making it possible to study a local charge environment.

Generally, all nuclei with spin $I>1 / 2$ have a nonspherical charge distribution and an electric quadrupole moment $Q$ associated with this charge. The quadrupole moment interacts with the local crystal electric-field gradient (EFG) that originates from a nonsymmetric ionic surrounding. The interaction of $Q$ with the EFG can be expressed by the following Hamiltonian [33]:

$$
\mathcal{H}_{\mathcal{Q}}=\frac{e^{2} q Q}{4 I(2 I-1)}\left[3 \hat{I}_{z}^{2}-\hat{I}^{2}+\frac{\eta}{2}\left(\hat{I}_{+}^{2}+\hat{I}_{-}^{2}\right)\right] .
$$

Here, $e$ is the charge of the proton and $\eta$ is the asymmetry parameter defined as $\eta=\frac{V_{x x}-V_{y y}}{V_{z z}}$. The $V_{i i}$ is the matrix element of the EFG tensor in the principal coordinate system. The quantity $q$ is commonly defined as $e q=V_{z z}$, and $\hat{I}_{z}, \hat{I}_{+}$, and $\hat{I}_{-}$are projections of the nuclear spin $I$ on the $z$-quantization axis given by the EFG and perpendicular to it, respectively. In $\mathrm{InCu}_{2 / 3} \mathrm{~V}_{1 / 3} \mathrm{O}_{3}$, the ${ }^{115}$ In nucleus with the spin $I=9 / 2$ is surrounded by six oxygen ions forming an asymmetric octahedron. Due to this asymmetry, the degeneracy of the NQR transitions $\pm 1 / 2 \leftrightarrow \pm 3 / 2, \pm 3 / 2 \leftrightarrow \pm 5 / 2, \pm 5 / 2 \leftrightarrow$ $\pm 7 / 2$, and $\pm 7 / 2 \leftrightarrow \pm 9 / 2$ is lifted and, as a result, four lines in the ${ }^{115}$ In NQR spectrum with different frequencies $v_{Q i}$ are expected (see below and the Appendix for details).

A full ${ }^{115} \mathrm{In} \mathrm{NQR}$ spectrum of $\mathrm{InCu}_{2 / 3} \mathrm{~V}_{1 / 3} \mathrm{O}_{3}$ measured at a high temperature of $T=100 \mathrm{~K}$ is presented in Fig. 1. Seven resonance lines can be resolved instead of the expected four.

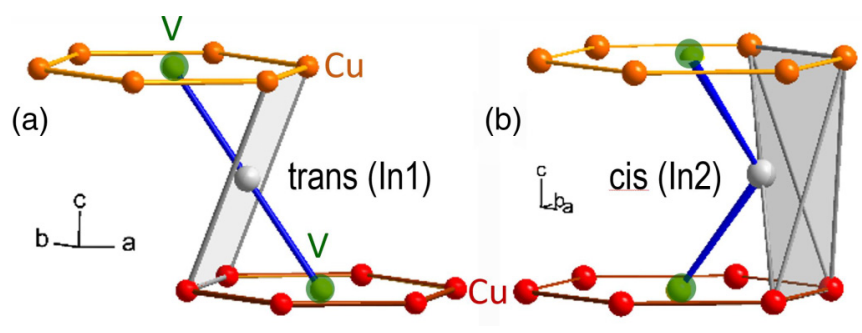

FIG. 2. Two crystallographic In1 and In2 positions in $\mathrm{InCu}_{2 / 3} \mathrm{~V}_{1 / 3} \mathrm{O}_{3}$ with (a) high and (b) low local environment symmetry, respectively.

Such a spectrum can be completely understood and well modeled if one takes into account two different crystallographic In positions, In1 and In2, present in the regular crystallographic structure of $\mathrm{InCu}_{2 / 3} \mathrm{~V}_{1 / 3} \mathrm{O}_{3}$ (Fig. 2). In1 occurs in a centrosymmetric surrounding in a trans-fashion, whereas In2 occurs in a noncentrosymmetric surrounding in a cis-fashion, thus having slightly different electric-field gradients.

Consequently, for the modeling of the spectra with the aid of Hamiltonian (1), we used two set of parameters $v_{Q}$ and $\eta$ which, together with the corresponding transition frequencies, are listed in Table I. Obviously, the smaller $\eta=0.05$ can be assigned to the position with higher symmetry and the larger $\eta=0.1$ can be assigned to the position with lower symmetry (Fig. 2). The intensities of the two contributions are related approximately as 1:1. The apparent difference of the amplitude of the lines in Fig. 1 is due to their slightly different widths, most likely occurring because of the different degree of distortion of the oxygen octahedron.

In the following, we focus on the part of the ${ }^{115}$ In NQR spectrum corresponding to the $\left( \pm \frac{3}{2} \leftrightarrow \pm \frac{5}{2}\right)$ transition, which is plotted on an enlarged scale in Fig. 3 for two selected temperatures of 100 and $37 \mathrm{~K}$. The spectrum measured in the paramagnetic state at $100 \mathrm{~K}$ consists of a single narrow line at $17.1 \mathrm{MHz}$ due to an almost perfect overlap of the signals from the In1 and In2 sites for the $\left( \pm \frac{3}{2} \leftrightarrow \pm \frac{5}{2}\right)$ transition (see Table I). The other spectrum recorded just below the ordering temperature $T^{* *}=39 \mathrm{~K}$ is significantly broader and is split into several lines. As can be seen in Fig. 4 on a larger frequency scale, with further decreasing temperature the splitting of the spectrum progresses and the lines get more resolved.

Typically, a splitting of an NQR line of a magnetic material is a fingerprint of an ordered state. If the probed nuclei are located at nonsymmetric positions with respect to the AFM sublattices, the nuclei are exposed to local static magnetic fields which cause the Zeeman splitting of the nuclear

TABLE I. Modeling parameters of the ${ }^{115}$ In NQR spectrum ( $v_{Q}$ and transition frequencies are given in $\mathrm{MHz}$ ).

\begin{tabular}{lcccccc}
\hline \hline & \multicolumn{5}{c}{ Transition frequencies } \\
\cline { 2 - 7 } Site & $v_{Q}$ & $\eta$ & $\left( \pm \frac{1}{2}, \pm \frac{3}{2}\right)$ & $\left( \pm \frac{3}{2}, \pm \frac{5}{2}\right)$ & $\left( \pm \frac{5}{2}, \pm \frac{7}{2}\right)$ & $\left( \pm \frac{7}{2}, \pm \frac{9}{2}\right)$ \\
\hline In1 & 8.58 & 0.05 & 8.77 & 17.09 & 25.72 & 34.31 \\
In2 & 8.66 & 0.1 & 9.51 & 17.08 & 25.92 & 34.60 \\
\hline \hline
\end{tabular}




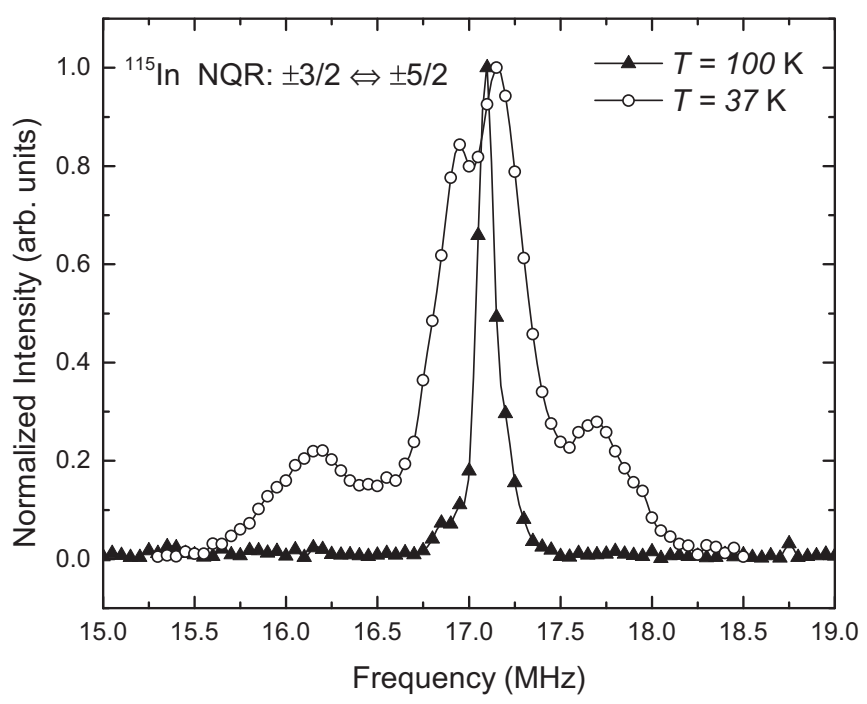

FIG. 3. ${ }^{115}$ In NQR spectra corresponding to the $\left( \pm \frac{3}{2} \leftrightarrow \pm \frac{5}{2}\right)$ transition (cf. Fig. 1) at temperatures of $100 \mathrm{~K}$ (triangles) and $37 \mathrm{~K}$ (circles).

Kramers doublets (see the Appendix for details). In the symmetrical case, the local field from different sublattices would be compensated and the NQR line should remain unsplit. When the spin system is in a static but not long-range ordered, i.e., disordered state, the individual nuclei in the sample would sense different local fields, leading to strong line broadening due to a distribution of local fields [34].

The splitting of the ${ }^{115} \mathrm{In}$ NQR lines evidences the establishment of the ordered state in $\mathrm{InCu}_{2 / 3} \mathrm{~V}_{1 / 3} \mathrm{O}_{3}$, where In nuclei are exposed to local magnetic fields giving rise to magnetically nonequivalent positions at each of the two crystallographic In sites. Apparently, those In nuclei which experience a stronger local field contribute to the lines with a

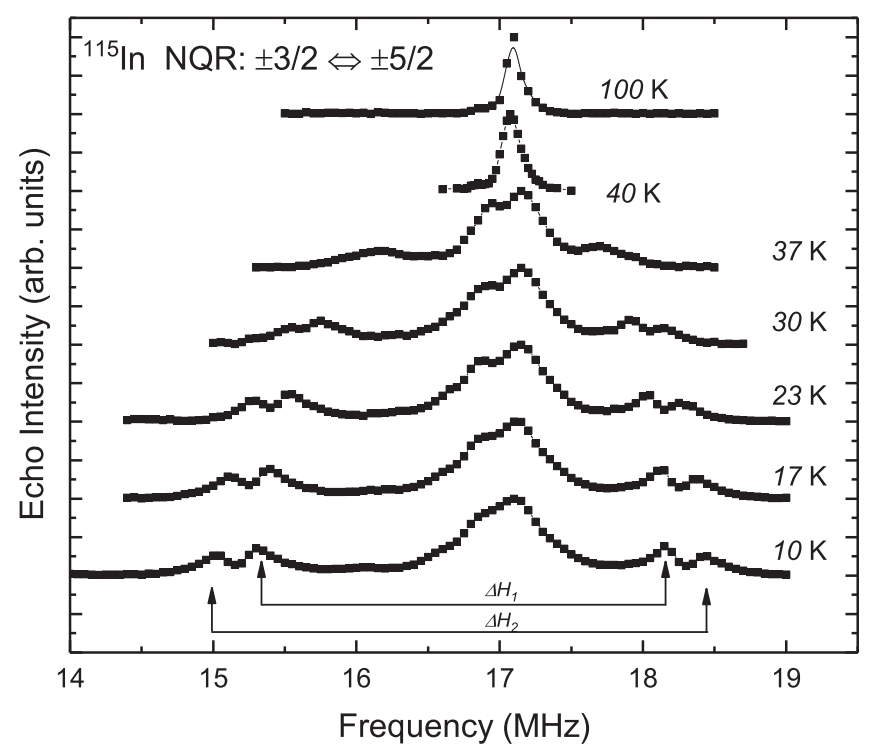

FIG. 4. Temperature dependence of the ${ }^{115}$ In NQR spectra corresponding to the $\left( \pm \frac{3}{2} \leftrightarrow \pm \frac{5}{2}\right)$ transition. Arrows labeled $\Delta H_{1}$ and $\Delta H_{2}$ indicate pairs of lines split by a strong internal magnetic field (see the text for details).

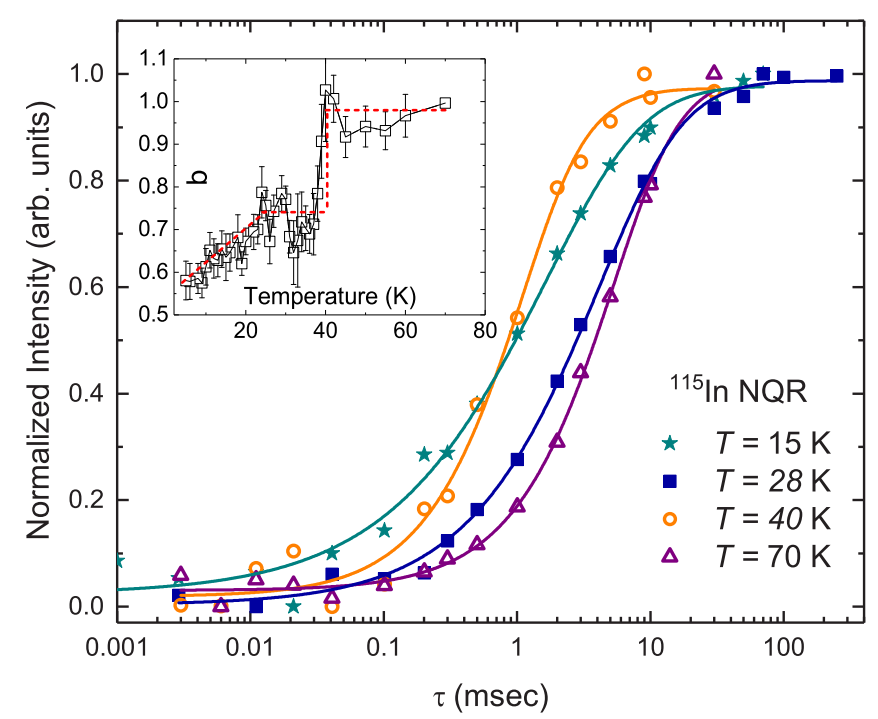

FIG. 5. Decay of the echo intensity as a function of the time delay $\tau$ between the pulses at temperatures of $70 \mathrm{~K}$ (triangles), $40 \mathrm{~K}$ (circles), $20 \mathrm{~K}$ (squares), and $15 \mathrm{~K}$ (stars). The inset depicts the $T$ dependence of the stretching parameter $b$ (see the text for details).

large splitting, as indicated in Fig. 4 by arrows labeled $\Delta H_{1}$ and $\Delta H_{2}$, whereas nuclei experiencing weaker magnetic fields contribute to the split lines close to the central frequency of $17 \mathrm{MHz}$ (see the Appendix).

\section{2. ${ }^{115}$ In relaxation measurements}

${ }^{115}$ In nuclear spin-lattice relaxation $\left(T_{1}^{-1}\right)$ measurements at the line corresponding to the $\pm 3 / 2 \leftrightarrow \pm 5 / 2$ transition were performed in a temperature range from 70 down to $5 \mathrm{~K}$. In Fig. 5, typical nuclear magnetization recovery curves obtained with the saturation-recovery pulse sequence are presented.

For nuclei with spin $I>3 / 2$, the magnetization recovery is a multiexponential function, but still can be described by a single relaxation time $T_{1}$. For $I=9 / 2$, the theoretical magnetization recovery curve contains four exponents. The corresponding coefficients of the exponential terms were calculated by Chepin and Ross Jr. for the relaxation in magnetic materials as a function of the asymmetry parameter $\eta$ in Ref. [35]. Since in $\mathrm{InCu}_{2 / 3} \mathrm{~V}_{1 / 3} \mathrm{O}_{3}$ two ${ }^{115} \mathrm{In}$ sites contribute to the total signal, the following function, which takes into account the overlap of the two signal contributions, was used to fit the data:

$$
\begin{aligned}
M(t) & =M_{0}\left[1-\left(A M_{\mathrm{In} 1}+B M_{\mathrm{In} 2}\right)\right], \\
M_{\mathrm{In} i} & =\sum_{k} C_{k} \exp \left[-\left(\frac{\rho_{k} t}{T_{1}}\right)^{b}\right] .
\end{aligned}
$$

Here, $M_{0}$ is the equilibrium magnetization, $T_{1}$ is the nuclear spin-lattice relaxation time, and $b$ is the stretching parameter. Parameters $A$ and $B$ are the weighting factors of the initial nuclear magnetization after the first pulse corresponding to the In 1 and In 2 sites, respectively. Since for the transition $\pm 3 / 2 \leftrightarrow \pm 5 / 2$ contributions from the two nuclear sites fully overlap, these parameters were taken equal, $A=B=0.5$. The corresponding numerical coefficients of Eq. (2) used to fit 
TABLE II. Relaxation exponents $\rho_{k}$ and corresponding coefficients $C_{k}$ for the $\left( \pm \frac{3}{2} \leftrightarrow \pm \frac{5}{2}\right)$ transition taken from Ref. [35] for $\eta=0.05$ (In1 site) and 0.1 (In2 site).

\begin{tabular}{lcccc}
\hline \hline Site & $\rho_{1}$ & $\rho_{2}$ & $\rho_{3}$ & $\rho_{4}$ \\
\hline In1 & 3 & 10 & 20.7 & 35.5 \\
In2 & 3 & 10 & 20.5 & 34 \\
Site & $C_{1}$ & $C_{2}$ & $C_{3}$ & $C_{4}$ \\
\hline In1 & 0.016 & 0.031 & 0.135 & 0.818 \\
In2 & 0.007 & 0.028 & 0.122 & 0.843 \\
\hline \hline
\end{tabular}

the magnetization recovery curves for both ${ }^{115}$ In positions are given in Table II.

The obtained temperature dependence of the nuclear spinlattice relaxation rate $T_{1}^{-1}$ is shown in Fig. 6. Generally, $T_{1}^{-1}$ can be driven by magnetic fluctuations and/or by a fluctuating EFG due to lattice vibrations (phonons). The latter mechanism yields $T_{1}^{-1}$ as a monotonically ascending function of temperature, e.g., $\propto T^{2}$ or $T^{7}$ [36], which is not observed experimentally (Fig. 6). Furthermore, in Ref. [25], three characteristic vibrational modes were observed with temperatures 160, 350, and $710 \mathrm{~K}$ corresponding to the lattice vibrations at $\mathrm{THz}$ frequencies, i.e., far above the NQR frequency scale. Thus, such phonon modes should not affect the nuclear relaxation processes as well. Therefore, we conclude that the nuclear relaxation is of magnetic origin at low temperatures. Indeed, a gradual increase of the $T_{1}^{-1}$ rate below $60 \mathrm{~K}$ (Fig. 6) can be ascribed to the development of the magnetic correlations in $\mathrm{InCu}_{2 / 3} \mathrm{~V}_{1 / 3} \mathrm{O}_{3}$. Eventually, the $T_{1}^{-1}(T)$ dependence exhibits a peak at temperature $T^{\star \star}=39 \mathrm{~K}$. Such a sharp peak usually signifies the establishment of long-range magnetic order. This peak in the relaxation is accompanied by a steplike drop of the stretching parameter $b$, indicating a distribution of the relaxation times due to inhomogeneous fluctuating fields in the electron spin system (Fig. 5, inset). Remarkably, by further decreasing the temperature, the second prominent feature,

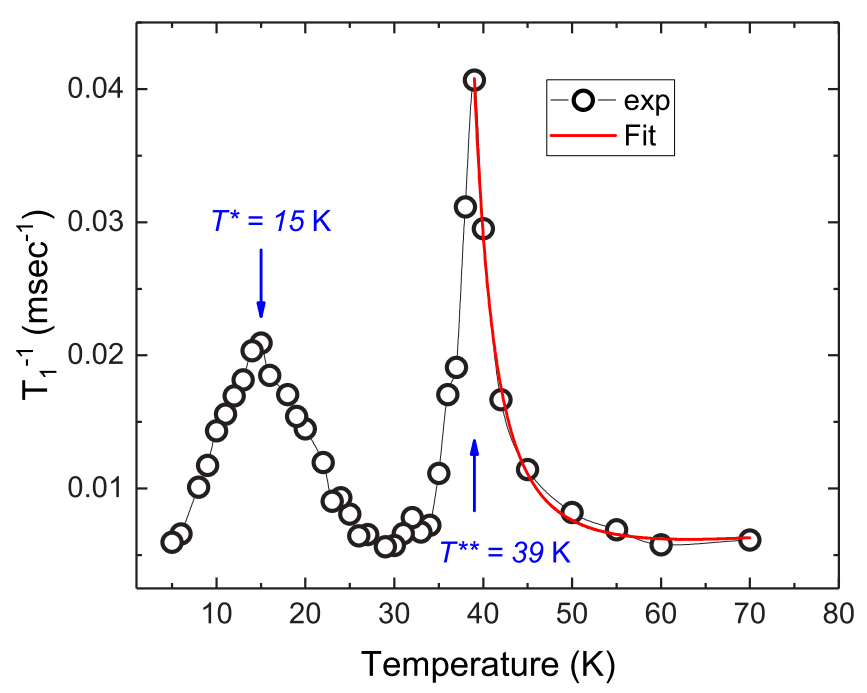

FIG. 6. Temperature dependence of the ${ }^{115}$ In spin-lattice relaxation rate $T_{1}^{-1}$ measured at the $\pm \frac{3}{2} \leftrightarrow \pm \frac{5}{2}$ transition.

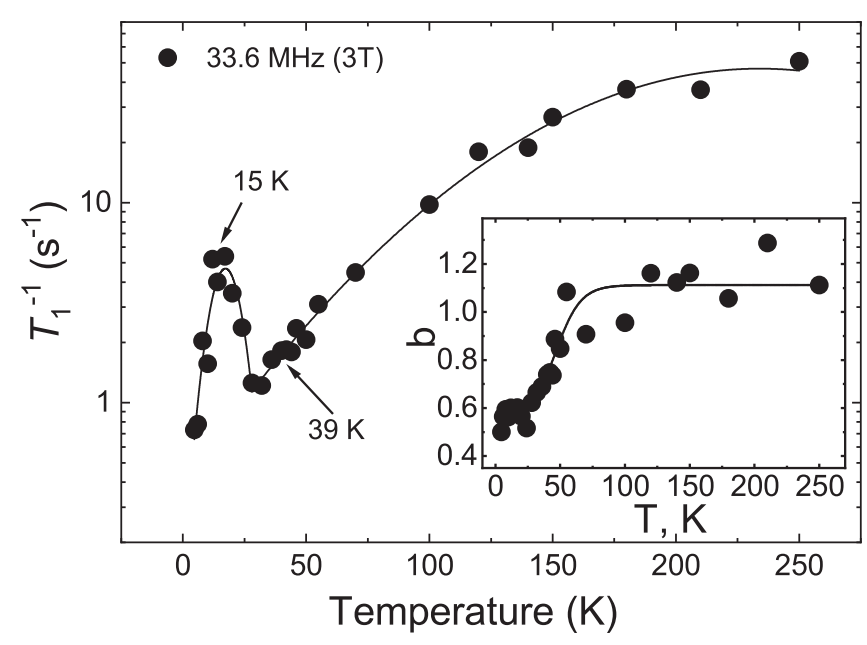

FIG. 7. Temperature dependence of the ${ }^{51} \mathrm{~V}$ spin-lattice relaxation rate measured in a magnetic field of $3 \mathrm{~T}$ (symbols). Characteristic temperatures $T^{*}=15 \mathrm{~K}$ and $T^{* *}=39 \mathrm{~K}$ are indicated by the arrows (cf. Fig. 6). Inset: $T$ dependence of the stretching parameter $b$ of the decay of the nuclear magnetization (symbols). Solid lines are guides for the eye (see the text for details).

a broad peak in the $T_{1}^{-1}(T)$ dependence at $T^{\star}=15 \mathrm{~K}$, is observed.

\section{B. ${ }^{51}$ V NMR spin-lattice relaxation rate}

The ${ }^{51} \mathrm{~V}$ NMR spin-lattice relaxation rate ${ }^{51} T_{1}^{-1}$ in $\mathrm{InCu}_{2 / 3} \mathrm{~V}_{1 / 3} \mathrm{O}_{3}$ was measured at the central peak of the ${ }^{51} \mathrm{~V}$ NMR spectrum [28] in magnetic fields of about $3 \mathrm{~T}$. The time evolution of the nuclear magnetization $M(t)$ could be described with a single spin-lattice relaxation time $T_{1}$ in the functional form described by Narath [37] for the central transition for the nuclear spin $I=5 / 2$. With decreasing temperature, ${ }^{51} T_{1}^{-1}(T)$ continuously decreases down to $\sim(35) \mathrm{K}$ and then exhibits a broad peak centered around $\sim(15) \mathrm{K}$ similar to the peak at $T^{*}=15 \mathrm{~K}$ in the $T$ dependence of the ${ }^{115}$ In NQR relaxation rate (cf. Fig. 6). Notably, in contrast to the ${ }^{115} \mathrm{In}$ NQR data, no peak at $T^{* *}=39 \mathrm{~K}$ was found in the $T$ dependence of the ${ }^{51} \mathrm{~V}$ NMR relaxation rate. The inset of Fig. 7 depicts the dependence of the stretching parameter $b$ of the nuclear magnetization decay on temperature. $b$ is close to unity at high temperatures and, similar to the case of the ${ }^{115} \mathrm{In}$ NQR relaxation (cf. Fig. 5, inset), rapidly decreases down to $b \sim 0.5$ below $40 \mathrm{~K}$.

\section{C. $\mu$ SR experiments}

\section{Transverse field $\mu \mathrm{SR}$ measurements}

The results of the transverse field (TF) $\mu$ SR measurements in $5 \mathrm{mT}$ external field are depicted in Fig. 8.

At $50 \mathrm{~K}$, the full muon spin polarization amplitude is oscillating with a frequency corresponding to the external field. A weak depolarization is due to a weak static field distribution at the muon site of the order of $10 \mathrm{G}$ caused by nuclear dipole moments. At $35 \mathrm{~K}$, the amplitude of this signal is reduced to $5 \%$, and $95 \%$ of the signal shows a much faster depolarization due to the appearance of strong 


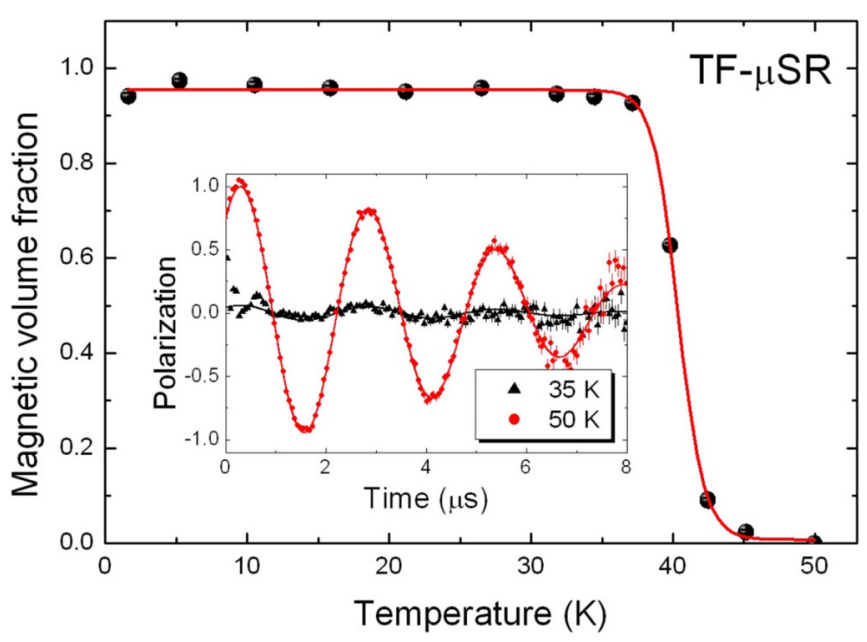

FIG. 8. Transverse field $\mu \mathrm{SR}$ results of $\mathrm{InCu}_{2 / 3} \mathrm{~V}_{1 / 3} \mathrm{O}_{3}$. Temperature dependence of the magnetic volume fraction determined from the amplitude fraction of muon spins precessing with the Larmor frequency $\omega_{\mu}=\gamma_{\mu} B_{\text {ext }}$ corresponding to the external field. The solid line is a guide for the eye. Inset: Typical time-dependent muon spin polarization at $60 \mathrm{~K}$ (red dots) and at $35 \mathrm{~K}$ (black triangles). The solid lines are results of a least-squares nonlinear fit analysis of the data as described in the text.

internal magnetic fields at the muon site which exceed the external field by more than a factor of 10 . The zero-field $\mu$ SR measurements discussed below prove that these internal fields are static on the timescale of microseconds. The muon spin polarization $P(t)$ in the TF time spectra was analyzed using the polarization function

$$
P(t)=P_{0}\left[1-f_{\text {mag }}(T)\right] \cos \left(\omega_{\mu} t+\phi\right) \exp \left(-\sigma_{\mathrm{TF}} t\right)^{2} .
$$

Here, $f_{\mathrm{mag}}(T)$ is the fraction of muons experiencing a strong internal magnetic field due to magnetic order, $\phi$ is the initial phase of the muon spin polarization at $t=0$, and $\sigma_{\mathrm{TF}}$ is the static Gaussian linewidth at $60 \mathrm{~K}$. Figure 8 shows $f_{\mathrm{mag}}(T)$. For temperatures below $40 \mathrm{~K}, f_{\text {mag }}(T)$ exhibits a nearly constant value of 0.95 . The remaining precession signal amplitude can be associated with muons stopped in the sample holder. Therefore, the TF $\mu \mathrm{SR}$ measurements prove that muons in the full sample volume experience strong static internal fields below $40 \mathrm{~K}$.

\section{Zero-field $\mu \mathrm{SR}$ measurements}

Zero-field (ZF) $\mu$ SR measurements were performed between 2 and $42.5 \mathrm{~K}$. The typical time spectra are depicted in Fig. 9.

At $42.5 \mathrm{~K}$, the muon spin polarization decays only weakly with a Gaussian time dependence. The relaxation rate $\sigma_{\mathrm{ZF}}$ is consistent with the TF measurements at high temperatures. At $40.2 \mathrm{~K}$, the onset of strong relaxation of a fraction of the full signal amplitude sets in. At $1.6 \mathrm{~K}$, two different spontaneous precession frequencies can be resolved: A weakly relaxing component with frequency $v_{1} \approx 2.5 \mathrm{MHz}$ and strongly relaxing component with $v_{2} \approx 8 \mathrm{MHz}$. A fraction of $f_{\text {long }} \approx$ $1 / 3$ of the initial muon spin polarization does show only a weak relaxation in the microsecond time range without oscillations. This proves that (i) two different muon sites are

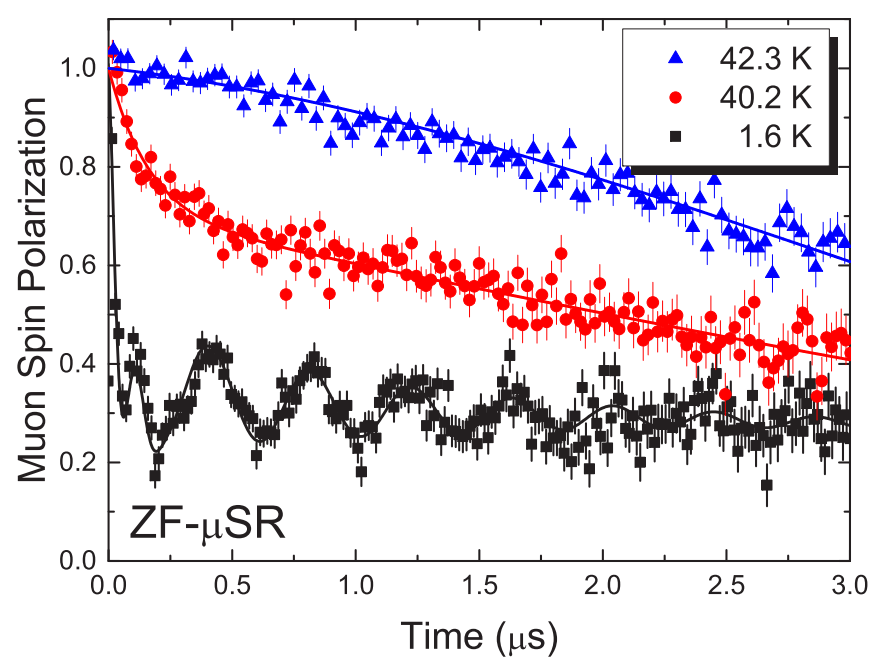

FIG. 9. Typical zero-field $\mu$ SR time spectra of $\operatorname{InCu}_{2 / 3} \mathrm{~V}_{1 / 3} \mathrm{O}_{3}$ at 42.3 K (blue triangles), 40.2 K (red dots), and 1.6 K (black squares). The solid lines are results of a least-squares nonlinear fit analysis of the data as described in the text.

populated in $\mathrm{InCu}_{2 / 3} \mathrm{~V}_{1 / 3} \mathrm{O}_{3}$, most likely due to $\mathrm{OH}$-like bonds of the muon to different oxygen ions in the lattice which are energetically nearly degenerated, and (ii) the internal fields at both muon sites are nearly static. Therefore, the ZF muon spin polarization $P(t)$ below $40 \mathrm{~K}$ was analyzed using the polarization function

$$
\begin{aligned}
P(t)= & P_{0}\left(f_{\text {long }} \exp \left(-\lambda_{1} t\right)+\left(1-f_{\text {long }}\right) \exp \left(-\sigma_{\mathrm{ZF}} t\right)^{2}\right. \\
& \times\left\{f_{1}(T) \cos \left[\omega_{1}(T) t\right] \exp \left[-\lambda_{\mathrm{t}, 1}(T) t\right]\right. \\
& \left.\left.+\left[1-f_{1}(T)\right] \cos \left[\omega_{2}(T) t\right] \exp \left[-\lambda_{\mathrm{t}, 2}(T) t\right]\right\}\right) .
\end{aligned}
$$

Here, $f_{1}(T)$ is the relative fraction of muon site 1 exhibiting the lower muon spin precession frequency $v_{1}=\omega_{1} / 2 \pi \approx$ $2.5 \mathrm{MHz}$ with the exponential relaxation rate $\lambda_{\mathrm{t}, 1}$, and $\nu_{2}=$ $\omega_{2} / 2 \pi \approx 8 \mathrm{MHz}$ with the exponential relaxation rate $\lambda_{\mathrm{t}, 2}$ being the corresponding parameters of muon site 2 . The relative signal amplitude of muon site $1, f_{1}(T) \approx 0.25$, is temperature independent below $30 \mathrm{~K}$. Above $30 \mathrm{~K}$, it increases towards 0.6 close to $40 \mathrm{~K}$. Experimentally, it was difficult to follow the high-frequency $\left(v_{2}\right)$ signal close to $T_{\mathrm{N}}$ due to its high relaxation rate. In the final analysis, we set the value of $f_{1}(T)=0.25$ constant for all temperatures $T<40 \mathrm{~K}$ since it is rather unlikely for the muon site occupancy ratio to change below $100 \mathrm{~K}$ in this material.

In Fig. 10, the temperature dependence of both muon spin precession frequencies is shown. In a global fit, $v_{1}$ and $v_{2}$ are very well described by an order parameter function of the form

$$
v_{i}(T)=v_{i, 0}\left(1-T / T_{\mathrm{N}}\right)^{\beta} \quad(i=1,2) .
$$

Here, $T_{\mathrm{N}}=39.4(1) \mathrm{K}$ and the critical exponent $\beta=$ $0.254(5)$ are global parameters, and $v_{1}=2.58(1) \mathrm{MHz}$ and $v_{2}=7.99$ (1) MHz. Clearly both curves show no anomaly near $T^{*}=15 \mathrm{~K}$, whereas $T_{\mathrm{N}}$ is identified with $T^{* *}=39 \mathrm{~K}$ (cf. Fig. 6).

The longitudinal relaxation rate $\lambda_{1}$, depicted in Fig. 11, exhibits a maximum close to $T_{\mathrm{N}}$. Below $38 \mathrm{~K}$, a slow decrease 


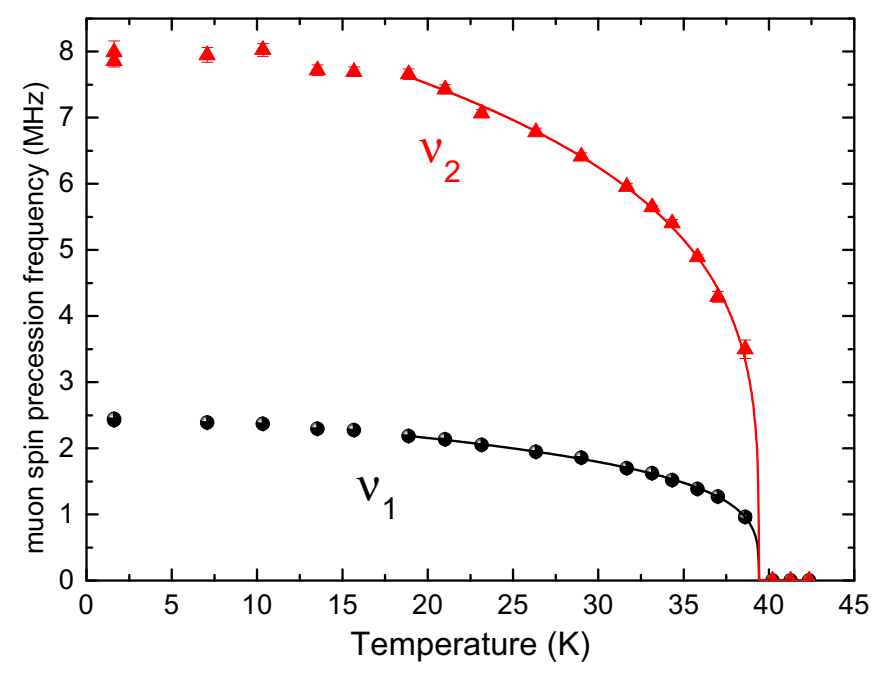

FIG. 10. Temperature dependence of the spontaneous muon spin precession frequencies $v_{1}$ (black dots) and $v_{2}$ (red triangles) in the magnetically ordered phase of $\mathrm{InCu}_{2 / 3} \mathrm{~V}_{1 / 3} \mathrm{O}_{3}$ below $40 \mathrm{~K}$. The global fit using an order parameter function given by Eq. (4) with $T_{\mathrm{N}}=39.4(1) \mathrm{K}$ and $\beta=0.254(5)$ is described in the text.

is observed from 0.07 towards $0.03 \mu \mathrm{s}^{-1}$ at $2 \mathrm{~K}$. Only a weak second maximum is found between 10 and $15 \mathrm{~K}$, reminiscent of the broad peak observed in $T_{1}^{-1}$ of ${ }^{115} \mathrm{In} \mathrm{NQR}$ at $T^{*}=$ $15 \mathrm{~K}$. The temperature shift of the peak position in the $\mu \mathrm{SR}$ experiment is consistent with the fact that the spontaneous muon spin precession frequencies are smaller than the NQR transition frequency by a factor of $\approx 2-6$ depending on the muon site.

The essentially static linewidths $\lambda_{\mathrm{t}, 1}$ and $\lambda_{\mathrm{t}, 2}$ are depicted in Fig. 12 (note that $\lambda_{1}$ is 1-2 orders of magnitude smaller). Since the local magnetic field at site 2 is $\approx 4$ times larger than at site $1, \lambda_{t, 2}$ is also larger than $\lambda_{t, 1}$ by a similar factor. Above

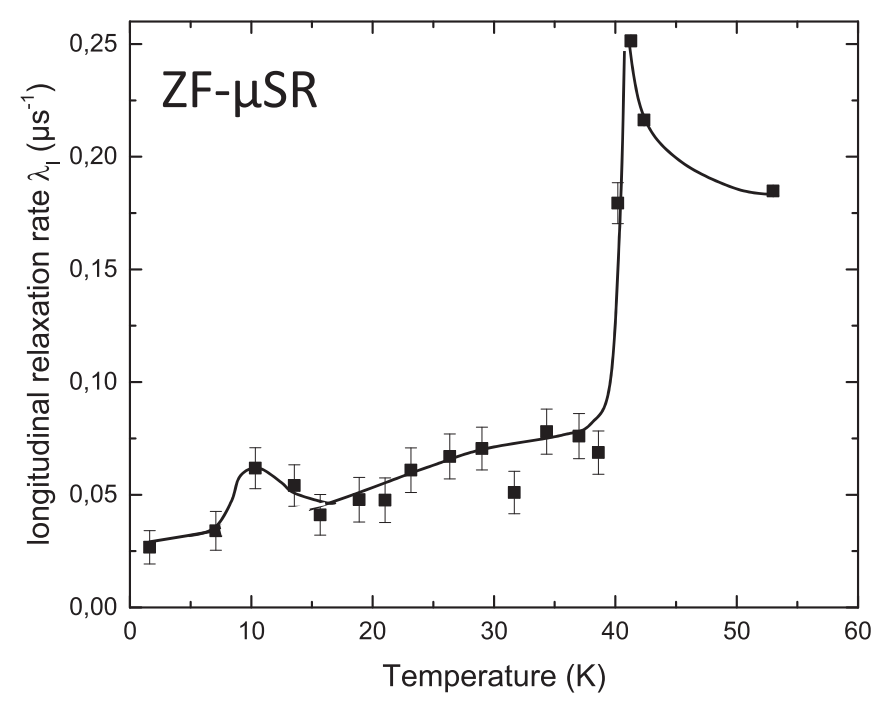

FIG. 11. Temperature dependence of the longitudinal relaxation rate $\lambda_{1}$ in $\mathrm{InCu}_{2 / 3} \mathrm{~V}_{1 / 3} \mathrm{O}_{3}$. In the magnetically ordered phase below $40 \mathrm{~K}$, in general, a weak monotonous decrease of $\lambda_{1}$ is observed superimposed by a small peak at $\approx(10) \mathrm{K}$. The solid and dashed lines are guides to the eye.

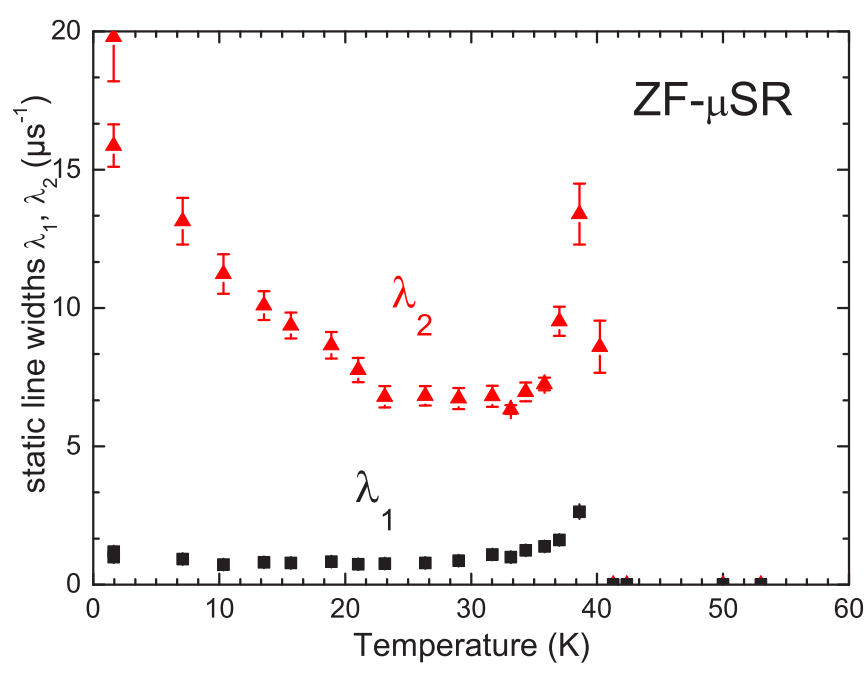

FIG. 12. Temperature dependence of the static linewidths $\lambda_{\mathrm{t}, 1}$ (black dots) and $\lambda_{\mathrm{t}, 2}$ (red triangles) in the magnetically ordered phase of $\mathrm{InCu}_{2 / 3} \mathrm{~V}_{1 / 3} \mathrm{O}_{3}$ below $40 \mathrm{~K}$.

$35 \mathrm{~K}$, towards $T_{\mathrm{N}}$, both linewidths increase due to the reduction of the in-plane magnetic coherence length close to $T_{\mathrm{N}}$. Below $30 \mathrm{~K}, \lambda_{\mathrm{t}, 1}$ is nearly temperature independent, whereas $\lambda_{t, 2}$ increases towards lower temperatures. This increase in linewidth may result from the onset of the static interlayer correlations in this temperature range.

\section{MICROSCOPIC MODEL}

Scalar relativistic DFT calculations have been performed within the generalized gradient approximation (GGA) [38] as implemented in the full potential code FPLO version 18 [39]. Nonmagnetic band structure calculations were performed on a $14 \times 14 \times 7 k$ mesh (228 points in the irreducible wedge). For the structural input, we used the neutron powder diffraction data measured at $10 \mathrm{~K}$ [25]. Note that the lattice constants and atomic coordinates provided in Table I of Ref. [25] pertain to the $\mathrm{Cu} / \mathrm{V}$ disordered structure with the space group $P 6_{3} / m m c$ (194) and cannot describe the honeycomb lattice structure of $\mathrm{InCu}_{2 / 3} \mathrm{~V}_{1 / 3} \mathrm{O}_{3}$. The highest symmetry compatible with the honeycomb arrangement of $\mathrm{Cu}$ atoms for a single layer is $P \overline{6} 2 m$ (189). Yet it features a simple stacking of magnetic honeycomb planes without a shift, giving rise to the spurious trigonal prismatic local coordination of In. Thus, the minimal structural model of $\mathrm{InCu}_{2 / 3} \mathrm{~V}_{1 / 3} \mathrm{O}_{3}$ entails the $A-B-A-B$ stacking, where the $A$ and $B$ planes are shifted with respect to each other, leading to the space group $\mathrm{Cmcm}$ (63).

The lack of a threefold rotation symmetry in the orthorhombic $\mathrm{Cmcm}$ structure singles out one of the three nearest-neighbor exchanges, which is no longer equivalent to the remaining two, and raises the question of a possible dimerization. To estimate this tendency, we relaxed the oxygen positions within the GGA $+U$ with $U_{d}=8.5 \mathrm{eV}$ and $J_{d}=1 \mathrm{eV}$ using the fully localized limit as the doublecounting correction. To allow for an antiferromagnetic arrangement within the honeycomb planes, the symmetry has been further lowered down to monoclinic, space group $P 2_{1} / \mathrm{m}$ (11). The resulting structure predictably has a lower GGA 

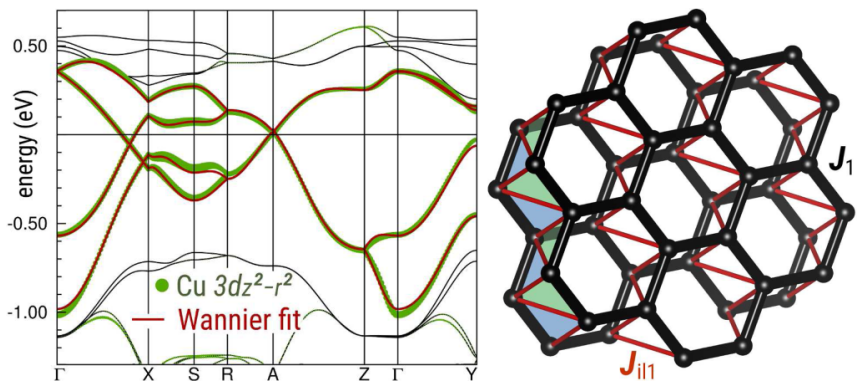

FIG. 13. Left panel: GGA band structure of $\mathrm{InCu}_{2 / 3} \mathrm{~V}_{1 / 3} \mathrm{O}_{3}$. The radii of the green circles denote the weight of the half-filled $\mathrm{Cu}$ $d_{3 z^{2}-r^{2}}$ orbital. Red solid lines are eigenvalues of the tight-binding Hamiltonian constructed from the Wannier projections. The notation of the $k$ points is $\Gamma=[0,0,0], X=\left[0, \frac{1}{3}, 0\right], S=\left[-\frac{1}{4}, \frac{1}{4}, 0\right]$, $R=\left[-\frac{1}{4}, \frac{1}{4}, \frac{1}{2}\right], A=\left[0, \frac{1}{3}, \frac{1}{2}\right], Z=\left[0,0, \frac{1}{2}\right]$, and $Y=\left[-\frac{1}{2}, 0,0\right]$ in terms of the reciprocal lattice vectors of the conventional ( $C$-centered) unit cell. Right panel: A sketch of the spin model of $\mathrm{InCu}_{2 / 3} \mathrm{~V}_{1 / 3} \mathrm{O}_{3}$ with the nearest-neighbor exchange $J_{1}$ (black cylinders) and the interlayer exchange $J_{\text {ill }}$ (red lines). Both exchanges are antiferromagnetic. Shaded triangles illustrate the magnetic frustration and are a guide to the eye. The spin model picture has been created using VESTA [48].

energy, but the difference between the transfer integrals for the inequivalent paths is small: $-174 \mathrm{meV}$ for the "singled out" path versus $-180 \mathrm{meV}$ for the other two paths. Moreover, the former transfer integral is smaller and the respective antiferromagnetic exchange is weaker, indicating that there is no tendency towards an electronically driven dimerization in the honeycomb planes. This also agrees with the experimental data where no fingerprints of a dimerization have been observed. Therefore, all further calculations were performed for the experimental crystal structure.

Though, in the chemical notation, $\mathrm{InCu}_{2 / 3} \mathrm{~V}_{1 / 3} \mathrm{O}_{3}$ is named indium copper oxide vanadate, from the physics perspective, electronically it is an undoped cuprate with the $3 d^{9}$ electronic configuration. Typical for this class of materials, GGA calculations yield a metallic ground state due to the severe underestimation of electronic correlations. The four $\mathrm{Cu}$ atoms in the unit cell produce a four-band manifold crossing the Fermi level (Fig. 13, left). In contrast to most cuprates that feature the half-filled $\mathrm{Cu} 3 d_{x^{2}-y^{2}}$ orbital, this band manifold in $\mathrm{InCu}_{2 / 3} \mathrm{~V}_{1 / 3} \mathrm{O}_{3}$ corresponds to the antibonding combination of $\sigma$-overlapping $\mathrm{Cu} 3 d_{3 z^{2}-r^{2}}$ and $\mathrm{O} 2 p_{z}$ orbitals. The strong hybridization allows us to resort to an effective single-orbital model with one orbital per $\mathrm{Cu}$. The transfer integrals $t_{i j}$ between these effective orbitals are estimated by Wannier projections [40]. In this way, we find that only three terms exceed $10 \mathrm{meV}$ : The first and second neighbors in the honeycomb plane $-t_{1}$ and $t_{2}$, respectively - as well as the shortest interlayer coupling $t_{\text {ill }}$.

The antiferromagnetic exchange can be directly estimated in second-order perturbation theory as $J_{i j}^{\mathrm{AF}}=4 t_{i j}^{2} / U_{\text {eff }}$, where $U_{\text {eff }}$ is the on-site Coulomb repulsion within the effective one-orbital model. By taking the commonly used value $U_{\text {eff }}=4.5 \mathrm{eV}$ (e.g., [41]), we obtain the leading antiferromagnetic exchange $J_{1}^{\mathrm{AF}}=360 \mathrm{~K}$, which is amenable to a direct comparison with the experiment: The magnetic susceptibility of the $S=\frac{1}{2}$ honeycomb Heisenberg model has
TABLE III. Transfer integrals $t_{i j}$ (in meV) of the effective oneorbital model and the respective magnetic exchanges $J_{i j}$ (in K) evaluated based on total-energy GGA $+U$ calculations. For each magnetic exchange, the respective interatomic $\mathrm{Cu}$... Cu distance (in $\AA$ ) and the multiplicity (within the unit cell) are provided.

\begin{tabular}{lcccr}
\hline \hline Path & $d_{\mathrm{Cu} \ldots \mathrm{Cu}}$ & Multiplicity & $t_{i j}$ & $J_{i j}$ \\
\hline$X_{1}$ & 3.3509 & 12 & -187 & 211.7 \\
$X_{2}$ & 5.8038 & 24 & -20 & -0.8 \\
$X_{\mathrm{il} 1}$ & 6.2572 & 16 & -49 & 5.4 \\
$X_{3}$ & 6.7018 & 12 & 6 & 0.3 \\
$X_{\mathrm{il} 2}$ & 7.0979 & 16 & 2 & -0.6 \\
\hline \hline
\end{tabular}

a broad maximum centered at $\sim 0.72 J_{1}$ (e.g., [42]). By taking the experimental position of this maximum (185 K [25]) in $\mathrm{InCu}_{2 / 3} \mathrm{~V}_{1 / 3} \mathrm{O}_{3}$, we obtain $J_{1}$ of about $255 \mathrm{~K}$. The reduced value of $J_{1}$ hints at a sizable ferromagnetic contribution to the magnetic exchange, which is lacking in the effective oneorbital approach in accord with the Pauli principle.

Next, we estimated the total exchange integrals, containing both antiferromagnetic and ferromagnetic contributions. To this end, we performed spin-polarized calculations, using a supercell doubled along the $a$ axis and calculated the total energies within the GGA $+U$ approach with the Coulomb repulsion $U_{d}=8.5 \mathrm{eV}$, the Hund's exchange $J_{d}=1.0 \mathrm{eV}$, and the fully localized limit [43] as the double-counting correction. All calculations have been done in the cell metrically equivalent to the unit cell doubled along the $a$ axis, the space group $P m$ (6), on a mesh of $2 \times 4 \times 4 k$ points. As expected, the GGA $+U$ restores the insulating nature of $\mathrm{InCu}_{2 / 3} \mathrm{~V}_{1 / 3} \mathrm{O}_{3}$, while the orbital occupation matrices indicate that the half-filled (and, hence, magnetically active) orbital in $\mathrm{InCu}_{2 / 3} \mathrm{~V}_{1 / 3} \mathrm{O}_{3}$ is $\mathrm{Cu} 3 d_{3 z^{2}-r^{2}}$.

The GGA $+U$ total energies of 31 different collinear magnetic configurations were mapped onto a classical Heisenberg model with $\left|\vec{S}_{i}\right|=\frac{1}{2}$. The five short-range (with $d_{\mathrm{Cu} . . \mathrm{Cu}}$ up to $7.5 \AA$ ) exchange integrals $J_{1}, J_{2}, J_{3}, J_{\text {ill }}$, and $J_{\text {il2 }}$ were determined by a least-squares solution to a redundant linear problem; the results are provided in the last column of Table III.

A corollary of this analysis is the presence of the dominant nearest-neighbor exchange $J_{1}$ and the much weaker interlayer exchange $J_{\text {il1 }}$ (see the right panel of Fig. 13 for a sketch of the model), while further exchange couplings are comparable to the error bars. At this point, it is crucial to consider the topology of the spin lattice. As we discussed earlier in this section, the neighboring layers in $\mathrm{InCu}_{2 / 3} \mathrm{~V}_{1 / 3} \mathrm{O}_{3}$ are shifted with respect to each other. Due to this shift, two interplane exchanges couple the nearest neighbors of one plane with the same spin in the neighboring plane. The resulting $J_{1}-J_{\text {ill }}-J_{\text {ill }}$ triangles underlie the geometrical frustration of the magnetic model (Fig. 13, right). We conclude that $\mathrm{InCu}_{2 / 3} \mathrm{~V}_{1 / 3} \mathrm{O}_{3}$ is an excellent realization of the honeycomb lattice where the magnetic ordering is further suppressed by a frustrated interlayer exchange.

Let us note that the absolute numerical values of $J_{1}$ and $J_{\text {il1 }}$ may be up to $15 \%$ inaccurate due to the ambiguous choice of the $U_{d}$ parameter. Yet their ratio $J_{\text {ill }} / J_{1} \simeq 2.5 \%$ and the irrelevance of further exchanges beyond the $J_{1}-J_{\text {ill }}$ model 
are a solid outcome of the analysis. It is clear that in the $\mathrm{J}_{\mathrm{il1}} \ll J_{1}$ regime, the anisotropy of $J_{1}$ may play a crucial role as its magnitude can be larger than the isotropic exchange $J_{\text {ill }}$. To investigate the anisotropic terms, we perform fullrelativistic noncollinear GGA $+U$ calculations using VASP version 5.4.4 [44] with projector augmented-wave pseudopotentials $[45,46]$. Anisotropic exchange parameters were evaluated by using the four-cell method [47]. The resulting bilinear exchange tensor $M_{1}$ is

$$
M_{1}=\left(\begin{array}{ccc}
239.4 & 0 & 0.7 \\
0 & 230.1 & 0 \\
-0.7 & 0 & 240.4
\end{array}\right) \mathrm{K} \text {, }
$$

where the honeycomb planes are in the $x z$ plane. Thus, the antisymmetric part of $M_{1}$ describes the DzyaloshinksiiMoriya vector $\left|D_{1}\right|=0.7 \mathrm{~K}$ perpendicular to the honeycomb planes. The extremely small value of $\left|D_{1}\right| / J_{1}$ of $\sim 0.3 \%$ again indicates that $\mathrm{InCu}_{2 / 3} \mathrm{~V}_{1 / 3} \mathrm{O}_{3}$ is an excellent model honeycomb system. In contrast, the diagonal elements reveal a considerable $X X Z$ anisotropy of $\sim 4 \%$.

The crystal field generated by the trigonal bipyramidal environment of copper atoms in $\mathrm{InCu}_{2 / 3} \mathrm{~V}_{1 / 3} \mathrm{O}_{3}$ renders the $\left|3 z^{2}-r^{2}\right\rangle$ orbital half filled and magnetically active. This unusual orbital ground state has been previously conjectured for two other cuprate materials: The spin chain system $\mathrm{CuSb}_{2} \mathrm{O}_{6}[49,50]$ and the 3D skyrmionic Mott insulator $\mathrm{Cu}_{2} \mathrm{OSeO}_{3}[51,52]$.

In $\mathrm{CuSb}_{2} \mathrm{O}_{6}$, the $\mathrm{CuO}_{6}$ octahedra are squeezed, forming two short and four long $\mathrm{Cu}-\mathrm{O}$ bonds. DFT calculations indicate a small crystal-field splitting, but the $\left|3 z^{2}-r^{2}\right\rangle$ orbital becomes half filled due to the larger bandwidth, which in turn gives rise to a sizable gain in kinetic energy [50]. Hence, the orbital ground state is stabilized by the competition between hopping processes and the on-site Coulomb repulsion rather than by the crystal field.

The case of $\mathrm{Cu}_{2} \mathrm{OSeO}_{3}$ is closer to $\mathrm{InCu}_{2 / 3} \mathrm{~V}_{1 / 3} \mathrm{O}_{3}$ : Here, one of the two structurally inequivalent copper atoms, $\mathrm{Cu}(1)$, has the local trigonal bipyramid environment. The microscopic magnetic model features five inequivalent exchanges, two ferromagnetic exchanges connecting $\mathrm{Cu}(2)$ atoms that have the conventional $\left|x^{2}-y^{2}\right\rangle$ orbital ground state, and three antiferromagnetic exchanges that couple $\mathrm{Cu}(1)$ and $\mathrm{Cu}(2)$ sublattices. Interestingly, one of these antiferromagnetic exchanges is accompanied by a large Dzyaloshinskii-Moriya anisotropy amounting to 58\% of the isotropic exchange [52]. However, in $\mathrm{InCu}_{2 / 3} \mathrm{~V}_{1 / 3} \mathrm{O}_{3}$, the situation is remarkably different: The $\left|3 z^{2}-r^{2}\right\rangle$ orbitals of the neighboring atoms are stretched perpendicular to the respective nearest-neighbor bonds, and the Dzyaloshinskii-Moriya anisotropy is nearly absent.

Coming back to the isotropic model, $\mathrm{InCu}_{2 / 3} \mathrm{~V}_{1 / 3} \mathrm{O}_{3}$ has a sizable nearest-neighbor superexchange, while longer-range intraplane exchanges $J_{2}$ and $J_{3}$ are strongly suppressed. As can be seen from the Wannier functions of $\left|3 z^{2}-r^{2}\right\rangle$ states in Fig. 14, this behavior can be understood as a joint effect of the $\left|3 z^{2}-r^{2}\right\rangle$ orbital state and the strong covalency of $\mathrm{V}-\mathrm{O}$ bonds. A sizable V-mediated superexchange is present in other low-dimensional magnets, such as volborthite $\mathrm{Cu}_{3} \mathrm{~V}_{2} \mathrm{O}_{7}(\mathrm{OH})_{2} \cdot 2 \mathrm{H}_{2} \mathrm{O}$ [53] and $\mathrm{SrNi}_{2} \mathrm{~V}_{2} \mathrm{O}_{8}$ [54].
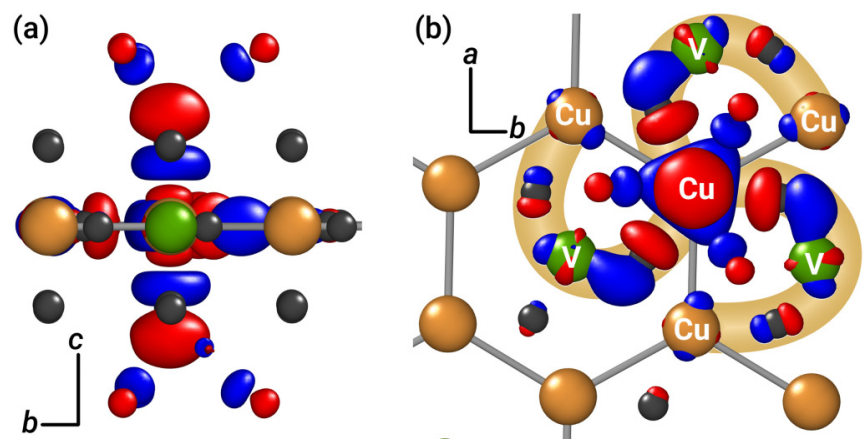

FIG. 14. Cu-centered Wannier functions for the $\left|3 z^{2}-r^{2}\right\rangle$ states in $\mathrm{InCu}_{2 / 3} \mathrm{~V}_{1 / 3} \mathrm{O}_{3}$ : (a) lateral and (b) top views. The $\mathrm{Cu}-\mathrm{O}-\mathrm{V}-\mathrm{O}-$ $\mathrm{Cu}$ paths facilitating a sizable antiferromagnetic $J_{1}$ exchange are highlighted in the right plot.

\section{DISCUSSION}

A very intriguing feature of $\mathrm{InCu}_{2 / 3} \mathrm{~V}_{1 / 3} \mathrm{O}_{3}$ is the occurrence of two characteristic temperatures in the low- $T$ regime of this compound. We begin the discussion by summarizing how these temperatures manifest in different kinds of measurements. The kink in the static magnetic magnetic susceptibility $\chi(T)$ at $38 \mathrm{~K}$ was originally ascribed to the 3D AFM order of the spins in the bulk of $\operatorname{InCu}_{2 / 3} \mathrm{~V}_{1 / 3} \mathrm{O}_{3}$ at $T_{\mathrm{N}}=38 \mathrm{~K}$ [27], and later on reconsidered to be a signature of local magnetic correlations of the spins at the structural domain boundaries [25]. Interestingly, the spin-flop transition in the field dependence of the static magnetization at $B_{\mathrm{sf}} \sim$ $5.8 \mathrm{~T}$ which usually occurs in $3 \mathrm{D}$ ordered anisotropic antiferromagnets is observed in $\mathrm{InCu}_{2 / 3} \mathrm{~V}_{1 / 3} \mathrm{O}_{3}$ only below $20 \mathrm{~K}$, which has been interpreted as the formation of the 3D Néeltype collinear AFM spin structure below this characteristic temperature [28]. This conclusion was corroborated by the occurrence of the second kink in the static susceptibility and by the observation of the gapped AFM resonance modes at sub-THz frequencies below $20 \mathrm{~K}$. Such resonance modes are typical for a 3D collinear antiferromagnet and soften at the same critical field $B_{\text {sf }}$ [28]. However, the gapless paramagnetic ESR signal can be observed only above the upper characteristic temperature of $38 \mathrm{~K}$ [27], suggesting that up to this temperature the spin system in $\mathrm{InCu}_{2 / 3} \mathrm{~V}_{1 / 3} \mathrm{O}_{3}$ still remains in some correlated state featuring anomalous spin dynamics [29].

Indeed, below the "upper" ordering temperature $T_{\mathrm{N}}=39 \mathrm{~K}$, as determined by the present local spin probe techniques, the development of the internal field-static on the timescale of the NQR and $\mu$ SR experiments-manifests in the splitting of the ${ }^{115}$ In NQR lines (Fig. 4) and in the spontaneous precession of the muon spin (Fig. 9). The splitting of the ${ }^{115}$ In NQR lines enables one to monitor the temperature dependence of the internal field $H_{\text {int }}$ probed by the nuclei which is proportional to the sublattice magnetization in $\mathrm{InCu}_{2 / 3} \mathrm{~V}_{1 / 3} \mathrm{O}_{3}$.

As to the dynamic characteristics, the $T_{1}^{-1}$ spin-lattice relaxation rate of ${ }^{115} \mathrm{In}$ nuclei exhibits a sharp peak at $T_{\mathrm{N}}=$ $T^{* *}=39 \mathrm{~K}$ and a broad peak at $T^{*}=15 \mathrm{~K}$ (Fig. 6), i.e., it is sensitive to both characteristic temperatures discussed above. The muon relaxation rate exhibits similar characteristic 

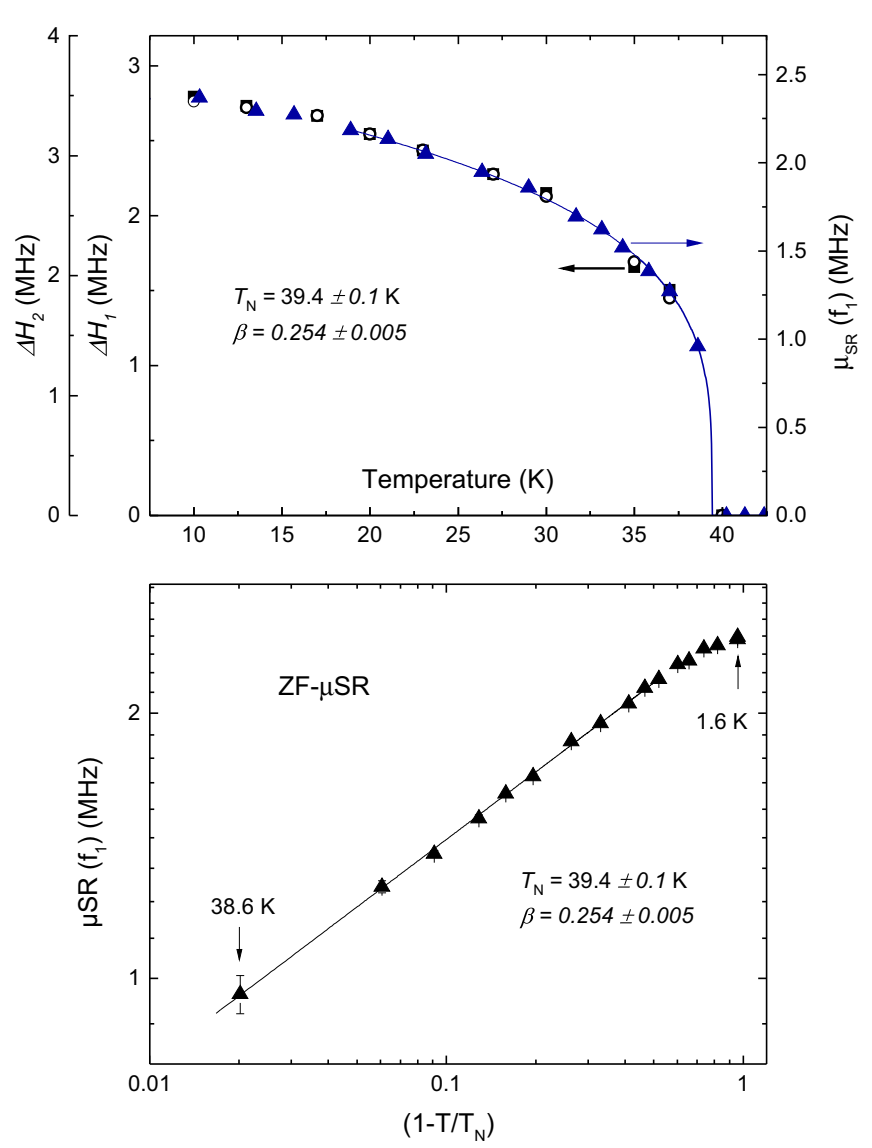

FIG. 15. Upper panel: ${ }^{115}$ In NQR line splitting (left scale) due to local internal magnetic field as a function of temperature [open circles correspond to the splitting $\Delta H_{1}$ and squares correspond to the splitting $\Delta H_{1}$ (cf. Fig. 4)] and $\mu_{S R}$ frequency (right scale) as a function of temperature (triangles). Lower panel: $\mu_{S R}$ frequency as a function of reduced temperature $\left(1-T / T_{\mathrm{N}}\right)$. The solid lines are the fit to the power-law function given by Eq. (4).

features (Fig. 11). In contrast, the $T_{1}^{-1}$ spin-lattice relaxation rate of ${ }^{51} \mathrm{~V}$ nuclei shows a peak only at $T^{*}=15 \mathrm{~K}$ (Fig. 7) due to a special symmetry position of these nuclei (see below).

The internal field $H_{\text {int }}$ probed by NQR and $\mu$ SR can be considered as a measure of the order parameter of the spin system in $\mathrm{InCu}_{2 / 3} \mathrm{~V}_{1 / 3} \mathrm{O}_{3}$. Therefore, it is instructive to analyze its temperature dependence in some detail. In NQR, as a quantity proportional to $H_{\text {int }}$, we have taken the full splitting of the outer satellites $\Delta H_{1}$ and $\Delta H_{2}$ (Fig. 4). Analysis of the $T$ dependence of $\Delta H_{i}$ can provide information about the spin dimensionality of the spin system. Such a dependence is shown in Fig. 15 together with the $T$ dependence of the muon precession frequency which perfectly match together. Both data sets were fitted together by the critical exponent function according to Eq. (4). The fit yields the ordering temperature $T_{\mathrm{N}}=39.4 \pm 0.1 \mathrm{~K}$ and the critical exponent $\beta=0.254 \pm 0.005$, the same as from the fit of the muon precession frequency alone (cf. Fig. 10).

The theoretically expected values of the critical exponent $\beta$ are $\beta=0.367$ for the 3D Heisenberg spin system, $\beta=0.345$ for the 3D $X Y$ model, $\beta=0.326$ for the 3D Ising system, $\beta=0.231$ for the $2 \mathrm{D} X Y$ model, and $\beta=0.125$ for the $2 \mathrm{D}$ Ising system [55-57]. The experimentally obtained value of $\beta=0.254$ is surprisingly close to the prediction for the $2 \mathrm{D}$ $X Y$ model [56], reflecting the predominantly two-dimensional critical behavior of quasistatically correlated $\mathrm{Cu}$ spins in $\mathrm{InCu}_{2 / 3} \mathrm{~V}_{1 / 3} \mathrm{O}_{3}$.

It appears from this analysis that although the occurrence of the internal field may be related to the development of 3D quasistatic correlations-as it follows from the quantum Monte-Carlo calculations in Ref. [28]-the correlations develop predominantly in the planes, whereas the interplane correlation length increases much slower. Furthermore, as it was shown in Ref. [56], in the framework of the 2D XY model, one finds finite magnetization at $T>0$ in 2D finite-size clusters, even if their size approaches a macroscopic scale. This situation is likely to be realized in $\mathrm{InCu}_{2 / 3} \mathrm{~V}_{1 / 3} \mathrm{O}_{3}$ since the finite in-plane structural correlation length of $\approx 300 \AA$ due to the $\mathrm{Cu} / \mathrm{V}$ site inversion [25] may set respective constraints on the magnetic correlation length. Therefore, one can consider the spin system in $\operatorname{InCu}_{2 / 3} \mathrm{~V}_{1 / 3} \mathrm{O}_{3}$ to be in a quasi-2D static state below $39 \mathrm{~K}$. As it follows from the microscopic model developed in Sec. IV, such an anisotropic behavior could be attributed to a significant geometrical frustration of the interlayer exchange due to the shift of the $\mathrm{Cu}-\mathrm{V}$ layers with respect to each other along the $c$ axis. Therefore, the $\mathrm{Cu}$ spins in the neighboring layers are AFM coupled on a frustrated triangular motif. In this situation, the planes are effectively decoupled but a small amount of defects can partially break the interlayer frustration, enabling some correlations also across the planes [58]. Moreover, as we have shown in Sec. IV, in $\mathrm{InCu}_{2 / 3} \mathrm{~V}_{1 / 3} \mathrm{O}_{3}$, there is a rather rare situation that the intralayer exchange, being much larger than that between the layers, is not frustrated, which stabilizes a quasistatic state below $T^{* *}=39 \mathrm{~K}$. Due to residual interlayer spin dynamics, this is not yet a conventional true 3D Néel AFM ordered state. This is evidenced by the pronounced 2D $X Y$ critical behavior and also by the absence of the fully developed AFM resonance modes and the field-induced spin-flop transition.

At first glance, it seems surprising that the slowing down of the in-plane spin dynamics in $\mathrm{InCu}_{2 / 3} \mathrm{~V}_{1 / 3} \mathrm{O}_{3}$ by approaching the upper characteristic temperature $T^{* *}=39 \mathrm{~K}$ from above does not result in a typical peak in the $T$ dependence of the ${ }^{51} \mathrm{~V}$ relaxation rate $T_{1}^{-1}$. However, one should keep in mind that the nonmagnetic $\mathrm{V}$ ions are located in the plane in the symmetric position with respect to the $\mathrm{Cu}$ ions. Therefore, the growth of the in-plane AFM correlations between the $\mathrm{Cu}$ spins results in a gradual decrease of the effective local field acting on the $\mathrm{V}$ nuclei ultimately down to zero. This yields the slowing down of the $T_{1}^{-1}$ rate despite the decreasing frequency of the spin fluctuations. This in-plane dynamics could be probed by $\mathrm{V}$ nuclei at the defect nonsymmetric crystallographic sites whose amount is, however, quite small in our samples. Nevertheless, the transition into a quasi-2D static state below $T^{* *}$ is reflected in a reduction of the stretching parameter $b$ from 1 to 0.5 , which is typical for a $2 \mathrm{D}$ situation (Fig. 7) [59]. In contrast, a characteristic peak at $T^{* *}$ is present in the $T_{1}^{-1}$ rate of ${ }^{115} \mathrm{In}$ nuclei which are in the nonsymmetric position with respect to the $\mathrm{Cu}$ spins in the plane (Fig. 2) and the parameter $b$ also drops down to 0.5 at $T^{* *}$ (Fig. 6). 


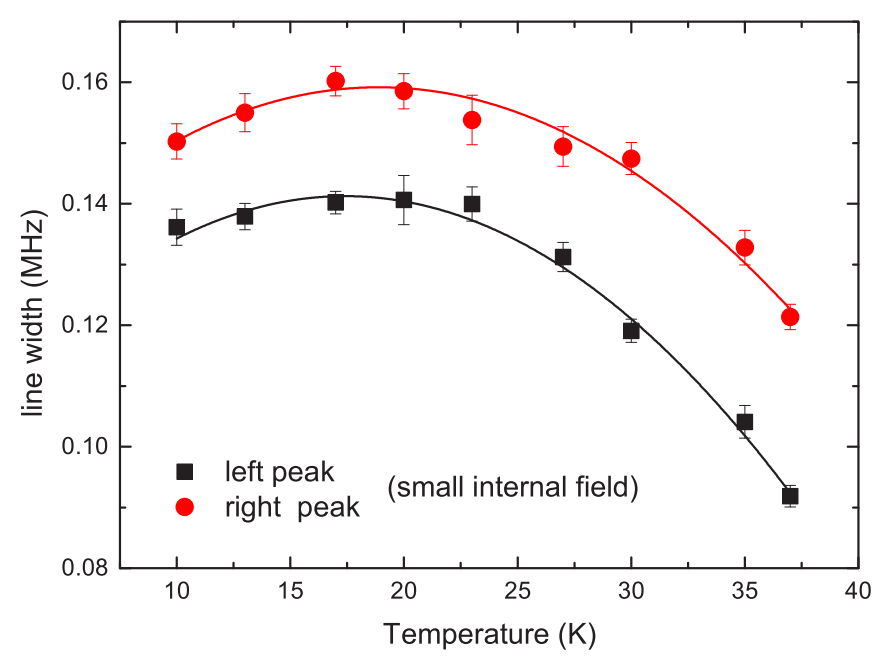

FIG. 16. $T$ dependence of the width of the left and right peaks in the central part of the ${ }^{115}$ In NQR spectrum (Fig. 3) corresponding to the In sites exposed to a small internal field. (Solid lines are guides for the eye.)

Similarly, the muons at the nonsymmetric interstitial position exhibit, at this temperature, a sharp peak in the relaxation rate too (Fig. 11).

The onset of the spin-flop transition below $\sim(20) \mathrm{K}$ [28] evidences the establishment of the fully developed 3D magnetic order in $\mathrm{InCu}_{2 / 3} \mathrm{~V}_{1 / 3} \mathrm{O}_{3}$. Due to the shift of the honeycomb planes along the $c$ axis, the local fields in the ordered state become nonzero at the $\mathrm{V}$ sites as well. Associated with this, the $T$ dependences of the $T_{1}^{-1}$ rate of both types of nuclei as well as the relaxation rate of the implanted muons feature the peak at $T^{*}=15 \mathrm{~K}$ (Figs. 6, 7, and 11). This peak is broad, suggesting a rather gradual transformation of the magnetic state obviously related to the strong interlayer frustration and the related residual interlayer spin dynamics, which is suppressed only gradually with decreasing temperature. The suppression of this dynamics and the establishment of the 3D order leads to a more homogeneous distribution of local fields. As a consequence, the NQR linewidth which continuously increased below $39 \mathrm{~K}$ saturates and even narrows below $\sim(20) \mathrm{K}$ (Fig. 16). The absence of an anomaly in the specific heat $C_{\mathrm{p}}(T)$ associated with magnetic order might be related to the fact that a large fraction of magnetic entropy is already lost below $100 \mathrm{~K}$ due to short-range correlations [25] as well as due to the pronounced two-dimensionality of the spin system which suppresses the $\lambda$ peak in the $C_{\mathrm{p}}(T)$ dependence at the magnetic phase transition [60]. Presumably, the ordered moment could be quite small due to the enhanced spin fluctuations in the $2 \mathrm{D}$ honeycomb lattice with the low coordination number $z=3$, which might explain the nonobservation of magnetic Bragg peaks in neutron diffraction [25]. The reason for a missing magnetic anomaly in the thermal expansion [25] could be a weak magnetoelastic coupling due to the quenched orbital moment of $\mathrm{Cu}^{2+}$.

The possibility of the realization of the BKT physics in $\mathrm{InCu}_{2 / 3} \mathrm{~V}_{1 / 3} \mathrm{O}_{3}$ is certainly a very intriguing issue. That the honeycomb planes in this compound feature planar anisotropy has been shown by our DFT calculations and previously was suggested by ESR experiments in Ref. [27]. It should be noted that for the occurrence of the BKT transition at a finite temperature, the pure $X Y$ limit is not necessary. As was shown by Cuccoli et al. [61] using quantum Monte Carlo simulations, even a very small (of the order of $10^{-3}$ ) deviation from the isotropic Heisenberg case towards planar anisotropy gives rise to the BKT transition at a finite temperature. As has been discussed, e.g., in Ref. [19], the occurrence of the Néel order obscures, but not necessarily completely excludes, the BKT physics. The detection of the BKT transition in low-dimensional quantum spin magnets is a general problem. Unlike in FM- or AFM-ordered phases, magnetization is not the order parameter for the BKT transition. Therefore, the signatures of the BKT physics in such experimental methods as neutron scattering, NMR, or ESR can be detected only indirectly by looking for critical exponents above the transition point. The critical behavior of the electron spin system can be studied by analyzing the $T$ dependence of the nuclear spin-lattice relaxation rate. Having established the 2D $X Y$ behavior below $T_{\mathrm{N}}=39 \mathrm{~K}$ from the analysis of the static internal field in $\mathrm{InCu}_{2 / 3} \mathrm{~V}_{1 / 3} \mathrm{O}_{3}$, one can expect that above this temperature, the correlation length $\xi(T)_{\mathrm{BKT}}$ follows an exponential dependence, predicted by Kosterlitz [18]:

$$
\xi(T)=\xi_{0} \exp \left(\frac{p}{\sqrt{T / T_{\mathrm{BKT}}-1}}\right),
$$

where $\xi_{0} \sim 1 \AA$ and $p \simeq \pi / 2 . T_{\mathrm{BKT}}$ is the BKT phase transition temperature into the vortex-antivortex paired state. As was shown by Borsa $e t$ al. [62], the spin-lattice relaxation rate in a $2 \mathrm{D}$ antiferromagnetic system is proportional to the square of the correlation length $\xi(T)$ :

$$
T_{1}^{-1}={ }^{115} \gamma^{2} \frac{h_{\mathrm{eff}}^{2}}{\omega_{e}}\left[\xi(T) / \xi_{0}\right]^{2} .
$$

Here, ${ }^{115} \gamma$ is the gyromagnetic factor of ${ }^{115} \mathrm{In}$, $\omega_{e}=\frac{J k_{B}}{\hbar} \sqrt{\frac{2 Z S(S+1)}{3}}$ is the exchange frequency, and $h_{\text {eff }}$ is the effective fluctuating hyperfine field [63]. Following the same procedure as used by Waibel et al. for the ${ }^{51} \mathrm{~V}$ NMR relaxation in $\mathrm{BaNi}_{2} \mathrm{~V}_{2} \mathrm{O}_{8}$ [21], the $\mathrm{NQR}$ magnetic spin-lattice relaxation rate for $\mathrm{InCu}_{2 / 3} \mathrm{~V}_{1 / 3} \mathrm{O}_{3}$ can be fitted with the following function:

$$
T_{1}^{-1}=A\left[\xi(T) / \xi_{0}\right]^{2}+k T,
$$

where $\xi(T)=\xi(T)_{\mathrm{BKT}}$, and the linear $T$ term accounts for the direct phonon relaxation. The fit yields $T_{\mathrm{BKT}}=32.5 \pm 1.8 \mathrm{~K}$ and $p=1.2 \pm 0.5$, which is close to the theoretical value $p \approx$ 1.6 within the error bar (Fig. 17).

Such an estimate of $T_{\mathrm{BKT}}$ appears to be consistent with the theoretical results in Ref. [56], where the temperature of the onset of the staggered magnetization (in our case, $T_{\mathrm{N}}=39 \mathrm{~K}$ ) of a 2D large-size spin cluster is somewhat higher than the BKT transition temperature.

From the theoretical perspective, our conjecture on the BKT transition in $\mathrm{InCu}_{2 / 3} \mathrm{~V}_{1 / 3} \mathrm{O}_{3}$ requires further analysis. Generally, a BKT transition should manifest itself in the behavior of long-range spin correlations at different temperatures. According to our DFT calculations, the minimal model to address the BKT physics in $\mathrm{InCu}_{2 / 3} \mathrm{~V}_{1 / 3} \mathrm{O}_{3}$ is the $S=\frac{1}{2} X X Z$ model of $A B$-stacked honeycomb lattices with 


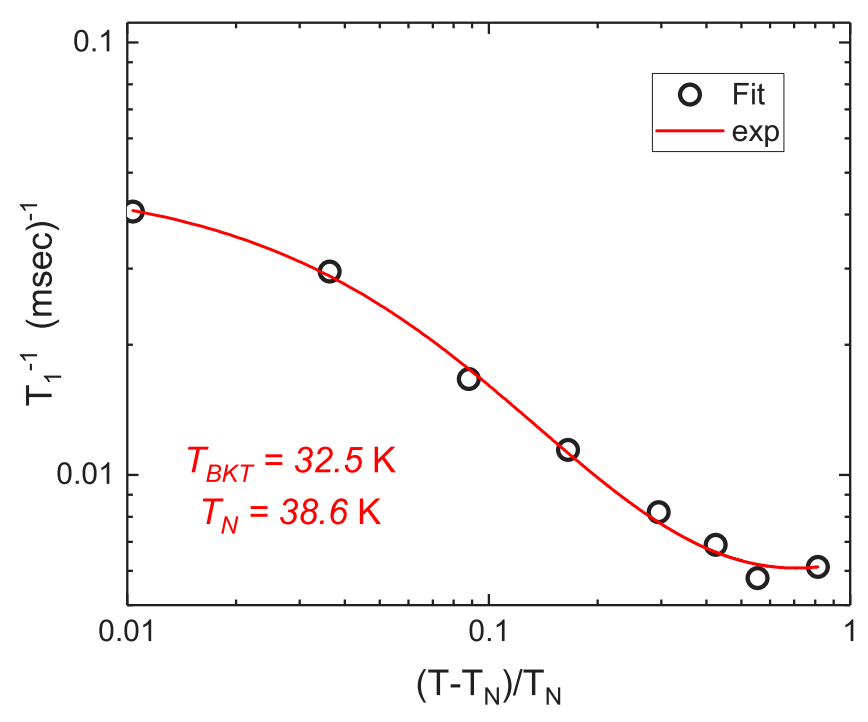

FIG. 17. ${ }^{115}$ In spin-lattice relaxation rate as a function of the reduced temperature $\left(T-T_{\mathrm{N}}\right) / T_{\mathrm{N}}$ (circles) and the fit according to Eq. (8) (solid line).

the interplane exchange amounting to $\sim 2.5 \%$ of the nearestneighbor exchange. Unfortunately, simulating this model is very challenging: While quantum Monte Carlo techniques suffer from the sign problem due to magnetic frustration, classical Monte Carlo simulations are not justified for the extreme quantum case of $S=\frac{1}{2}$. Nevertheless, we believe that our experimental indications of the BKT transition in

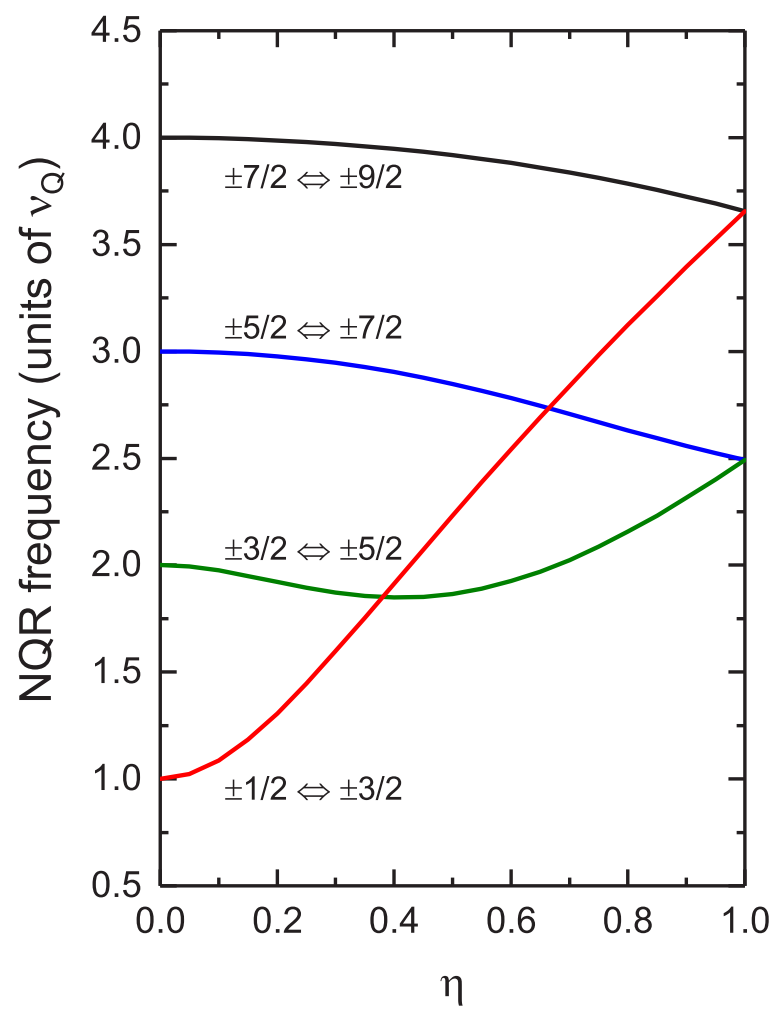

FIG. 18. NQR transition frequencies as a function of the EFG asymmetry parameter $\eta$ numerically calculated using Hamiltonian (1).
$\mathrm{InCu}_{2 / 3} \mathrm{~V}_{1 / 3} \mathrm{O}_{3}$ will stimulate further numerical studies of this model, for instance, using the recently introduced pseudofermion functional renormalization-group method [64], capable of treating quantum spin models with frustration.

\section{CONCLUSIONS}

To summarize, we have studied the low-temperature magnetic properties of the quasi-2D magnet $\mathrm{InCu}_{2 / 3} \mathrm{~V}_{1 / 3} \mathrm{O}_{3}$ featuring honeycomb planes of AFM coupled $\mathrm{Cu}^{2+}$ spins $S=1 / 2$ by employing three kinds of local spin probes: The nuclei ${ }^{115} \mathrm{In}$ and ${ }^{51} \mathrm{~V}$ at the regular lattice sites and implanted spinpolarized muons $\mu^{+}$at interstitial lattice sites. The main objective of this study was to elucidate the nature of the two characteristic temperature scales of $\sim 40$ and $\sim(20) \mathrm{K}$, which were controversially interpreted in different previous experiments. The splitting of the ${ }^{115}$ In NQR spectral lines and
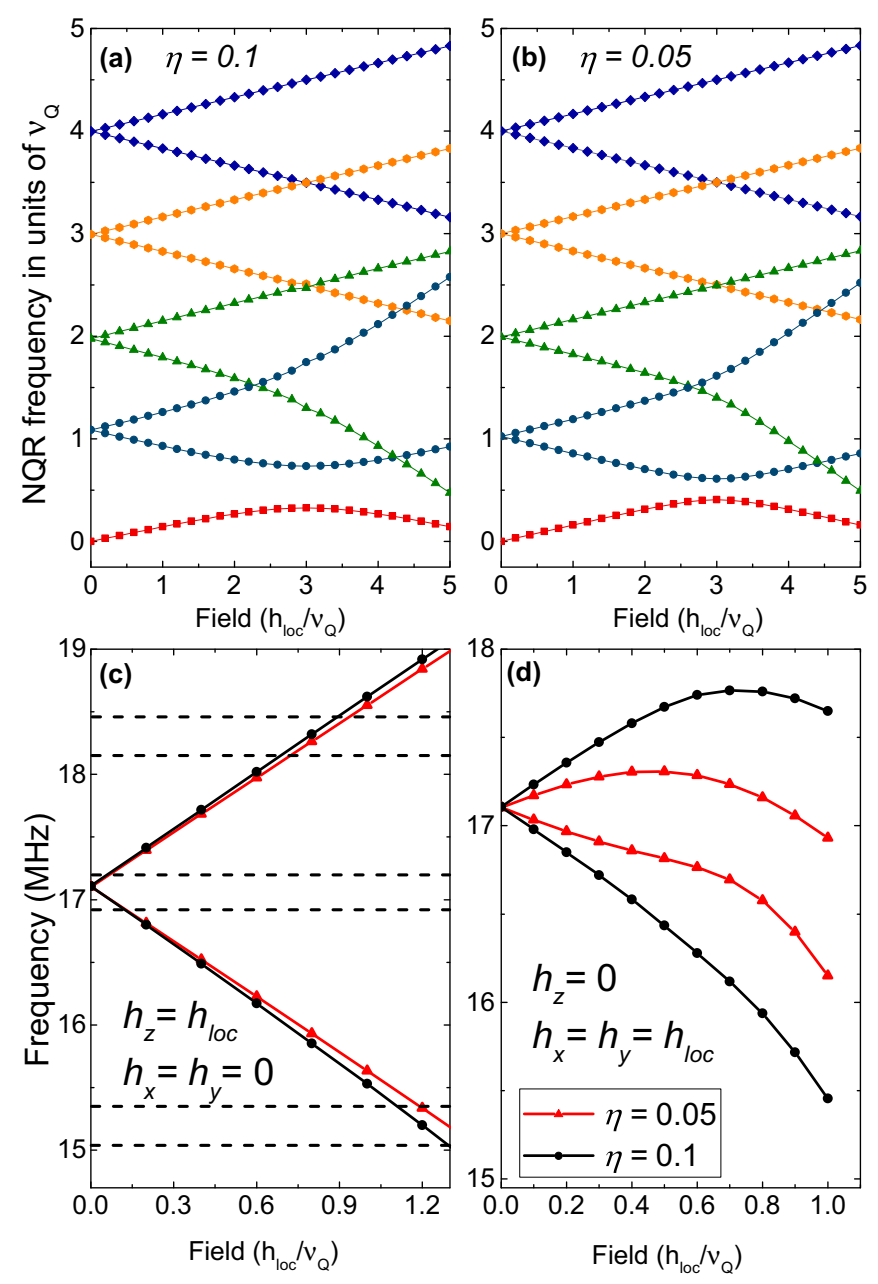

FIG. 19. (a), (b) NQR transition frequencies as a function of local magnetic field for the nuclear spin $I=\frac{9}{2}$ in an asymmetric electric field with (a) $\eta=0.1$ and (b) $\eta=0.05$. The magnetic field $h$ is applied along the $z$ symmetry axis of EFG. (c) Comparison of the calculated frequencies for the $\pm \frac{3}{2} \leftrightarrow \pm \frac{5}{2}$ transition for $h \| z$ with the position of the experimentally observed NQR signals at $T=$ $10 \mathrm{~K}$ indicated by horizontal dashed lines (cf. Fig. 4). (d) Calculated frequencies for $h \perp z$. Red solid triangles in (c) and (d) correspond to $\eta=0.05$ and black circles correspond to $\eta=0.1$. 
the onset of the muon spin precession at $39 \mathrm{~K}$ evidence the development of the staggered magnetization whose temperature dependence agrees well with the predictions of the $2 \mathrm{D}$ $X Y$ model, suggesting that $\mathrm{InCu}_{2 / 3} \mathrm{~V}_{1 / 3} \mathrm{O}_{3}$ is in a quasi-2D static magnetic state below this temperature. A transition to this state is signified by a peak in the $T$ dependence of the relaxation rate $T^{-1}$ of the In nuclei at $T^{* *}=39 \mathrm{~K}$ and a maximum in the longitudinal muon spin-relaxation rate, whereas such a peak is absent in the case of the $\mathrm{V}$ nuclei due to the cancellation of the local fluctuating fields at this symmetric in-plane position, as expected for the quasi-2D ordered state. However, a true 3D long-range magnetic order in $\mathrm{InCu}_{2 / 3} \mathrm{~V}_{1 / 3} \mathrm{O}_{3}$ gradually sets in at a significantly lower temperature, manifesting in the broad relaxation peaks at $T^{*}=15 \mathrm{~K}$ for both types of nuclei and a weak anomaly in the muon relaxation rate. These experimental results are strongly supported by our DFT calculations of the electronic band structure and of the exchange constants in $\mathrm{InCu}_{2 / 3} \mathrm{~V}_{1 / 3} \mathrm{O}_{3}$ that reveal the dominance of the nearest-neighbor AFM exchange in the honeycomb spin- $1 / 2$ planes with a significant $X X Z$ anisotropy and with negligible further neighbor in-plane couplings, as well as a single sizable and frustrated AFM exchange between the honeycomb planes. It appears from our experimental and theoretical findings that such a significant interlayer magnetic frustration concomitant with some structural disorder give rise to two distinct magnetic states successively occurring in $\mathrm{InCu}_{2 / 3} \mathrm{~V}_{1 / 3} \mathrm{O}_{3}$ upon lowering the temperature. Particularly intriguing are indications from the analysis of the ${ }^{115}$ In relaxation rate $T^{-1}(T)$ of the topological BKT transition in the honeycomb planes of $\mathrm{InCu}_{2 / 3} \mathrm{~V}_{1 / 3} \mathrm{O}_{3}$ in the quasi-2D ordered state presumably occurring at $T_{\mathrm{BKT}}=$ $33 \mathrm{~K}$, which call for theoretical studies of the BKT physics in this compound.

\section{ACKNOWLEDGMENTS}

This work has been supported in part by the Deutsche Forschungsgemeinschaft in the framework of CRC "Correlated Magnetism - From Frustration to Topology" (SFB 1143, Project ID No. 247310070) and Würzburg-Dresden Cluster of Excellence on Complexity and Topology in Quantum Matter ct.qmat (EXC 2147, Project ID No. 39085490), and is partially based on experiments performed at the Swiss Muon Source $\mathrm{S} \mu \mathrm{S}$, Paul Scherrer Institute, Villigen, Switzerland. E.V. and M.I. acknowledge the support of RFBR through Grant No. 18-02-00664. O.J. was supported by the Leibniz Association through the Leibniz Competition. A.M. acknowledges support by the Carl Zeiss Foundation. We thank Ulrike Nitzsche for technical assistance.

\section{APPENDIX}

Hamiltonian (1) can be easily solved for the asymmetry parameter $\eta=0$, i.e., in the case of the axial symmetry of the charge distribution around the nucleus. For $\eta \neq 0$, it is still solvable analytically for the nuclei with $I=3 / 2$, but for $I>3 / 2$ there is no analytical solution. Therefore, for ${ }^{115} \mathrm{In}$ with $I=9 / 2$, we have calculated the transition frequencies as a function of $\eta$ numerically.

The results for the four allowed transitions $\pm 1 / 2 \leftrightarrow \pm 3 / 2$, $\pm 3 / 2 \leftrightarrow \pm 5 / 2, \pm 5 / 2 \leftrightarrow \pm 7 / 2$, and $\pm 7 / 2 \leftrightarrow \pm 9 / 2$ are presented in Fig. 18. As is customary, the levels are labeled according to the largest component of the wave function, though $I_{z}$ is a good quantum number only when $\eta=0$.

Additionally, we numerically solved Hamiltonian (1) perturbed by the Zeeman interaction due to the local magnetic field $h$. For calculations, we considered two cases of the EFG with $\eta=0.05$ and $\eta=0.1$ relevant for $\mathrm{InCu}_{2 / 3} \mathrm{~V}_{1 / 3} \mathrm{O}_{3}$. Computation results are shown in Figs. 19(a) and 19(b). As a consequence of the Zeeman splitting, the additional $-\frac{1}{2} \leftrightarrow \frac{1}{2}$ transition appears. In our calculations, we considered the case when the magnetic field is applied along the EFG $z$-principal axis. A comparison of the calculations with the experimental data obtained at $10 \mathrm{~K}$ (cf. Fig. 4) is shown in Fig. 19(c). As can be seen there, in the case of very small magnetic fields, the line splitting is small and is masked by the overlap of the lines. In stronger fields, all four transitions are well separated, in qualitative agreement with experiment. On the quantitative level, there is some discrepancy due to the noncollinearity of $h$ and the $z$ axis of EFG, which results in an asymmetric splitting of the lines. To illustrate this, in Fig. 19(d) we show the other simple limit of $h \perp z\left(h_{x}=h_{y}\right)$.
[1] N. D. Mermin and H. Wagner, Phys. Rev. Lett. 17, 1133 (1966).

[2] E. A. Zvereva, M. I. Stratan, Y. A. Ovchenkov, V. B. Nalbandyan, J.-Y. Lin, E. L. Vavilova, M. F. Iakovleva, M. Abdel-Hafiez, A. V. Silhanek, X.-J. Chen, A. Stroppa, S. Picozzi, H. O. Jeschke, R. Valentí, and A. N. Vasiliev, Phys. Rev. B 92, 144401 (2015).

[3] A. I. Kurbakov, A. N. Korshunov, S. Y. Podchezertsev, A. L. Malyshev, M. A. Evstigneeva, F. Damay, J. Park, C. Koo, R. Klingeler, E. A. Zvereva, and V. B. Nalbandyan, Phys. Rev. B 96, 024417 (2017).

[4] A. K. Bera, S. M. Yusuf, A. Kumar, and C. Ritter, Phys. Rev. B 95, 094424 (2017).

[5] E. Lefrançois, M. Songvilay, J. Robert, G. Nataf, E. Jordan, L. Chaix, C. V. Colin, P. Lejay, A. Hadj-Azzem, R. Ballou, and V. Simonet, Phys. Rev. B 94, 214416 (2016).
[6] O. A. Petrenko, G. Balakrishnan, N. R. Wilson, S. de Brion, E. Suard, and L. C. Chapon, Phys. Rev. B 78, 184410 (2008).

[7] P. H. Y. Li, R. F. Bishop, D. J. J. Farnell, and C. E. Campbell, Phys. Rev. B 86, 144404 (2012).

[8] A. Mulder, R. Ganesh, L. Capriotti, and A. Paramekanti, Phys. Rev. B 81, 214419 (2010).

[9] H. Mosadeq, F. Shahbazi, and S. A. Jafari, J. Phys.: Condens. Matter 23, 226006 (2011).

[10] R. F. Bishop, P. H. Y. Li, D. J. J. Farnell, and C. E. Campbell, J. Phys.: Condens. Matter 24, 236002 (2012).

[11] A. Yogi, A. K. Bera, A. Maurya, R. Kulkarni, S. M. Yusuf, A. Hoser, A. A. Tsirlin, and A. Thamizhavel, Phys. Rev. B 95, 024401 (2017).

[12] R. F. Bishop, P. H. Y. Li, and C. E. Campbell, Phys. Rev. B 89, 214413 (2014). 
[13] O. Smirnova, M. Azuma, N. Kumada, Y. Kusano, M. Matsuda, Y. Shimakawa, T. Takei, Y. Yonesaki, and N. Kinomura, J. Am. Chem. Soc. 131, 8313 (2009).

[14] M. Matsuda, M. Azuma, M. Tokunaga, Y. Shimakawa, and N. Kumada, Phys. Rev. Lett. 105, 187201 (2010).

[15] V. L. Berezinskii, Sov. Phys. JETP-USSR 32, 493 (1971).

[16] J. M. Kosterlitz and D. J. Thouless, J. Phys. C: Solid State Phys. 6, 1181 (1973).

[17] J. M. Kosterlitz and D. J. Thouless, J. Phys. C: Solid State Phys. 5, L124 (1972).

[18] J. M. Kosterlitz, J. Phys. C: Solid State Phys. 7, 1046 (1974).

[19] L. P. Regnault and J. Rossat-Mignod, Phase transitions in quasi two-dimensional planar magnets, in Magnetic Properties of Layered Transition Metal Compounds, edited by L. J. de Jongh (Springer, Netherlands, 1990), pp. 271-321.

[20] M. Heinrich, H.-A. Krug von Nidda, A. Loidl, N. Rogado, and R. J. Cava, Phys. Rev. Lett. 91, 137601 (2003).

[21] D. Waibel, G. Fischer, T. Wolf, H. v. Löhneysen, and B. Pilawa, Phys. Rev. B 91, 214412 (2015).

[22] E. S. Klyushina, B. Lake, A. T. M. N. Islam, J. T. Park, A. Schneidewind, T. Guidi, E. A. Goremychkin, B. Klemke, and M. Månsson, Phys. Rev. B 96, 214428 (2017).

[23] H. Rønnow, A. Wildes, and S. Bramwell, Phys. B 276-278, 676 (2000).

[24] U. Tutsch, B. Wolf, S. Wessel, L. Postulka, Y. Tsui, H. O. Jeschke, I. Opahle, T. Saha-Dasgupta, R. Valentí, A. Brühl, K. Removic-Langer, T. Kretz, H.-W. Lerner, M. Wagner, and M. Lang, Nat. Commun. 5, 5169 (2014).

[25] A. Möller, U. Löw, T. Taetz, M. Kriener, G. André, F. Damay, O. Heyer, M. Braden, and J. A. Mydosh, Phys. Rev. B 78, 024420 (2008).

[26] S. qing Jia, Q. wei Wang, J. Liu, and L.-J. Zou, J. Phys.: Conf. Ser. 827, 012010 (2017).

[27] V. Kataev, A. Möller, U. Löw, W. Jung, N. Schittner, M. Kriener, and A. Freimuth, J. Magn. Magn. Mater. 290-291, 310 (2004).

[28] M. Yehia, E. Vavilova, A. Möller, T. Taetz, U. Löw, R. Klingeler, V. Kataev, and B. Büchner, Phys. Rev. B 81, 060414(R) (2010).

[29] S. Okubo, H. Wada, H. Ohta, T. Tomita, M. Fujisawa, T. Sakurai, E. Ohmichi, and H. Kikuchi, J. Phys. Soc. Jpn. 80, 023705 (2011).

[30] Y. J. Yan, Z. Y. Li, T. Zhang, X. G. Luo, G. J. Ye, Z. J. Xiang, P. Cheng, L. J. Zou, and X. H. Chen, Phys. Rev. B 85, 085102 (2012).

[31] S.-Q. Jia, Q.-W. Wang, X.-L. Yu, and L.-J. Zou, AIP Adv. 7, 055825 (2017).

[32] A. Suter and B. M. Wojek, Phys. Procedia 30, 69 (2012).

[33] A. Abragam, Principles of Nuclear Magnetism (Clarendon, Oxford, 1961).

[34] M. Iakovleva, S. Zimmermann, J. Zeisner, A. Alfonsov, H.-J. Grafe, M. Valldor, E. Vavilova, B. Büchner, and V. Kataev, Phys. Rev. B 96, 064417 (2017).
[35] J. Chepin and J. H. Ross, Jr., J. Phys.: Condens. Matter 3, 8103 (1991).

[36] J. V. Kranendonk, Physica 20, 781 (1954).

[37] A. Narath, Phys. Rev. 162, 320 (1967).

[38] J. P. Perdew, K. Burke, and M. Ernzerhof, Phys. Rev. Lett. 77, 3865 (1996).

[39] K. Koepernik and H. Eschrig, Phys. Rev. B 59, 1743 (1999).

[40] H. Eschrig and K. Koepernik, Phys. Rev. B 80, 104503 (2009).

[41] O. Janson, W. Schnelle, M. Schmidt, Y. Prots, S.-L. Drechsler, S. K. Filatov, and H. Rosner, New J. Phys. 11, 113034 (2009).

[42] A. A. Tsirlin, O. Janson, and H. Rosner, Phys. Rev. B 82, 144416 (2010).

[43] M. T. Czyżyk and G. A. Sawatzky, Phys. Rev. B 49, 14211 (1994).

[44] G. Kresse and J. Furthmüller, Phys. Rev. B 54, 11169 (1996); Comput. Mater. Sci. 6, 15 (1996).

[45] P. E. Blöchl, Phys. Rev. B 50, 17953 (1994).

[46] G. Kresse and D. Joubert, Phys. Rev. B 59, 1758 (1999).

[47] H. Xiang, C. Lee, H.-J. Koo, X. Gong, and M.-H. Whangbo, Dalton Trans. 42, 823 (2013).

[48] K. Momma and F. Izumi, J. Appl. Cryst. 44, 1272 (2011).

[49] M. Heinrich, H.-A. Krug von Nidda, A. Krimmel, A. Loidl, R. M. Eremina, A. D. Ineev, B. I. Kochelaev, A. V. Prokofiev, and W. Assmus, Phys. Rev. B 67, 224418 (2003).

[50] D. Kasinathan, K. Koepernik, and H. Rosner, Phys. Rev. Lett. 100, 237202 (2008).

[51] S. Seki, X. Z. Yu, S. Ishiwata, and Y. Tokura, Science 336, 198 (2012).

[52] O. Janson, I. Rousochatzakis, A. A. Tsirlin, M. Belesi, A. A. Leonov, U. K. Rößler, J. van den Brink, and H. Rosner, Nat. Commun. 5, 5376 (2014).

[53] O. Janson, S. Furukawa, T. Momoi, P. Sindzingre, J. Richter, and K. Held, Phys. Rev. Lett. 117, 037206 (2016).

[54] A. K. Bera, B. Lake, A. T. M. N. Islam, O. Janson, H. Rosner, A. Schneidewind, J. T. Park, E. Wheeler, and S. Zander, Phys. Rev. B 91, 144414 (2015).

[55] L. de Jongh, Magnetic Properties of Layered Transition Metal Compounds (Springer, Netherlands, 1990).

[56] S. T. Bramwell and P. C. W. Holdsworth, J. Phys.: Condens. Matter 5, L53 (1993).

[57] M. F. Collins, Magnetic Critical Scattering (Oxford University Press, New York, 1989).

[58] D.-Y. Liu, Y. Guo, X.-L. Zhang, J.-L. Wang, Z. Zeng, H.-Q. Lin, and L.-J. Zou, Europhys. Lett. 103, 47010 (2013).

[59] A. Narayanan, J. Hartman, and A. Bain, J. Magn. Reson., Ser. A 112, 58 (1995).

[60] P. Bloembergen, Physica B 85, 51 (1977).

[61] A. Cuccoli, T. Roscilde, V. Tognetti, R. Vaia, and P. Verrucchi, Phys. Rev. B 67, 104414 (2003).

[62] F. Borsa, M. Corti, T. Goto, A. Rigamonti, D. C. Johnston, and F. C. Chou, Phys. Rev. B 45, 5756 (1992).

[63] T. Moriya, Prog. Theor. Phys. 16, 23 (1956).

[64] Y. Iqbal, R. Thomale, F. Parisen Toldin, S. Rachel, and J. Reuther, Phys. Rev. B 94, 140408(R) (2016). 